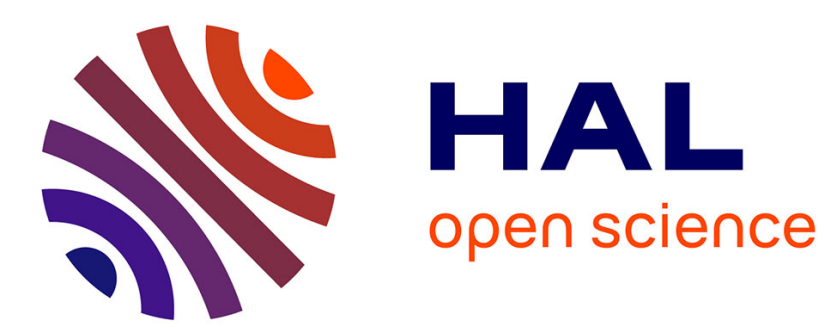

\title{
Nonlinear dynamics of rectangular plates: investigation of modal interaction in free and forced vibrations
}

\author{
Michele Ducceschi, Cyril Touzé, Stefan Bilbao, Craig J. Webb
}

\section{To cite this version:}

Michele Ducceschi, Cyril Touzé, Stefan Bilbao, Craig J. Webb. Nonlinear dynamics of rectangular plates: investigation of modal interaction in free and forced vibrations. Acta Mechanica, 2014, 225 (1), pp.213-232. 10.1007/s00707-013-0931-1 . hal-01134793

\section{HAL Id: hal-01134793 \\ https://hal-ensta-paris.archives-ouvertes.fr/hal-01134793}

Submitted on 24 Mar 2015

HAL is a multi-disciplinary open access archive for the deposit and dissemination of scientific research documents, whether they are published or not. The documents may come from teaching and research institutions in France or abroad, or from public or private research centers.
L'archive ouverte pluridisciplinaire HAL, est destinée au dépôt et à la diffusion de documents scientifiques de niveau recherche, publiés ou non, émanant des établissements d'enseignement et de recherche français ou étrangers, des laboratoires publics ou privés. 


\title{
Nonlinear dynamics of rectangular plates: investigation of modal interaction in free and forced vibrations
}

\author{
Michele Ducceschi $^{a} \cdot$ Cyril Touzé $^{a} \cdot$ Stefan Bilbao $^{b} \cdot$ Craig J. \\ Webb $^{b}$
}

the date of receipt and acceptance should be inserted later

\begin{abstract}
Nonlinear vibrations of thin rectangular plates are considered, using the von Kármán equations in order to take into account the effect of geometric nonlinearities. Solutions are derived through an expansion over the linear eigenmodes of the system for both the transverse displacement and the Airy stress function, resulting in a series of coupled oscillators with cubic nonlinearities, where the coupling coefficients are functions of the linear eigenmodes. A general strategy for the calculation of these coefficients is outlined, and the particular case of a simply supported plate with movable edges is thoroughly investigated. To this extent, a numerical method based on a new series expansion is derived to compute the Airy stress function modes, for which an analytical solution is not available. It is shown that this strategy allows the calculation of the nonlinear coupling coefficients with arbitrary precision, and several numerical examples are provided. Symmetry properties are derived to speed up the calculation process and to allow a substantial reduction in memory requirements. Finally, analysis by continuation allows an investigation of the nonlinear dynamics of the first two modes both in the free and forced regimes. Modal interactions through internal resonances are highlighted, and their activation in the forced case is discussed, allowing to compare the nonlinear normal modes (NNMs) of the undamped system with the observable periodic orbits of the forced and damped structure.
\end{abstract}

\section{Introduction}

Plates elements are commonly found in a variety of contexts in structural mechanics. An understanding of their vibrational properties is crucial in many contexts, e.g. fluid-structure interaction problems, plate and panel flutter in aeronautics [13], energy harvesting of fluttering flexible plates [18], piezoelectric and laminated plates [15,21], as well as their coupling with electro-magnetic and thermal fields [22]. When the plates are thin, vibration amplitudes can easily attain the same order of magnitude as the thickness. In this case the nonlinear geometric effects cannot be neglected, resulting in a rich variety of dynamics [38,2]. Examples can be given ranging from weakly to strongly nonlinear cases: nonlinear vibrations of plates with moderate nonlinearity [45,2], fluid-structure interaction problems [24], and the transition from periodic to chaotic vibrations $[37,4,50]$. Aside from typical engineering problems, the chaotic dynamics exhibited by thin plates excited at large amplitudes finds application in the field of musical acoustics, as it accounts for the bright and shimmering sound of gongs and cymbals $[28,12,7,6]$. It was pointed out recently, from the theoretical, numerical and experimental viewpoints, that the complex dynamics of thin plates vibrating at large amplitudes displays the characteristics of wave turbulence systems and thus it should be studied within this framework $[20,9,34,35,49]$.

A widely used model in nonlinear plate modeling is due to von Kármán [54]. This model takes into account a quadratic correction to the longitudinal strain, as compared to the classical linear plate equation by Kirchhoff [16,38,46, 33]. The type of nonlinearity introduced is thus purely geometrical. The von Kármán equations are particularly appealing because they describe a large range of phenomena while retaining a relatively compact form, introducing a single bilinear operator in the classic linear equations by Kirchhoff.

Pioneering analytical work in the analysis of rectangular thin plate vibrations with geometrical nonlinearities was carried out in the 1950s by Chu and Herrmann [17], demonstrating for the first time the hardening-type nonlinearity that has been confirmed by numerous experiments; see e.g. [27,1]. Restricting the attention to the case of rectangular plates, the work by Yamaki [55] confirms analytically the hardening-type nonlinearity for forced plates. The case of 1:1 internal resonance for rectangular plates (where two eigenmodes have nearly equal eigenfrequencies) has been studied by Chang et al. [14], and by Anlas and Elbeyli [3]. Parametrically excited nearly square plates, also displaying 1:1 internal resonance, have also been considered by Yang and Sethna [56]. All these works focus on the moderately nonlinear dynamics of rectangular plates where only a few modes (typically one or two) interact together. In these cases, the von Kármán plate equations are projected onto the linear modes and the coupling coefficients are computed with ad hoc assumptions that appear difficult to generalize. Finite element methods have also been employed-see e.g. the work by Ribeiro et al. $[42,43,44]$, and Boumediene et al. [10] to investigate the nonlinear forced response in the vicinity of a eigenfrequency.

${ }^{a}$ Unité de Mecanique, ENSTA - ParisTech, 828 Boulevard des Maréchaux, Palaiseau, France ${ }^{b}$ James Clerk Maxwell Building, University of Edinburgh, Scotland 
Recently, numerical simulations of more complex dynamical solutions, involving a very large number of modes in the permanent regime, have been conducted, in order to simulate the wave turbulence regime and to reproduce the typical sounds of cymbals and gongs. For that, Bilbao developed an energy-conserving scheme for finite difference approximation of the von Kármán system [5], which allows the study of the transition to turbulence [49] and the simulation of realistic sounds of percussive plates and shells $[7,6]$. Spectral methods with a very large number of degrees of freedom have also been employed in [20] to compare theoretical and numerical wave turbulence spectra.

This works aims at extending the possibilities of the modal approach to simulate numerically the nonlinear regime of rectangular plates. Instead of introducing ad-hoc assumptions, a general model is here presented; this model retains a vast number of interacting modes, making possible the investigation of the global dynamics of the plate while making it very precise. Within this framework the advantages of the modal approach are retained (accuracy of linear and nonlinear coefficients, flexibility in setting modal damping terms in order to calibrate simulation with experiment, ...) and its limitations are overcome: there is no restriction with respect to the amount of modes that one wants to keep. In this work the possibility of simulating dynamical solutions with a large number (say a few hundred) of modes is detailed. The case under study is that of a simply supported plate with in-plane movable edges. For this particular choice, the transverse modes are readily obtained from a double sine series [25]; the in-plane modes, however, are not available in closed form. Interestingly, it was shown in [46] that the problem of finding the in-plane modes for the chosen boundary conditions corresponds mathematically to the problem of finding the modes of a fully clamped Kirchhoff plate. To this extent, a general strategy proposed in [30] is here adapted to find the clamped plate modes. To validate the results, the resonant response of the plate in the vicinity of the first two modes is numerically investigated, for vibration amplitudes up to three to four times the thickness. Secondly, a thorough comparison of the modal approach with the finite difference method developed in $[5,6]$ is also given. Calculation of the the free response allows the study of the first two nonlinear normal modes of the plate, and to highlight the complicated dynamics displayed at large amplitudes. Modal couplings, resonant and non-resonant, are investigated. Finally the forced response is also computed and the link between the backbone curve and the forced response is investigated, showing the role of internal resonance and damping.

\section{Model Description}

Plates whose flexural vibrations are comparable to the thickness are most efficiently described by the von Kármán equations [39,17,46,33]. In the course of this paper, a rectangular plate of dimensions $L_{x}, L_{y}$ and thickness $h$ (with $\left.h \ll L_{x}, L_{y}\right)$ is considered. The plate material is homogeneous, of volume density $\rho$, Young's modulus $E$ and Poisson's ratio $v$. Its flexural rigidity is then defined as $D=E h^{3} / 12\left(1-v^{2}\right)$. The von Kármán system then reads

$$
\begin{aligned}
& D \Delta \Delta w+\rho h \ddot{w}+c \dot{w}=L(w, F)+\delta\left(\mathbf{x}-\mathbf{x}_{\mathbf{0}}\right) f \cos (\Omega t), \\
& \Delta \Delta F=-\frac{E h}{2} L(w, w),
\end{aligned}
$$

where $\Delta$ is the Laplacian operator, $w=w(x, y, t)$ is the transverse displacement and $F=F(x, y, t)$ is the Airy stress function. The equations present a viscous damping term $c \dot{w}$ and a sinusoidal forcing term $\delta\left(\mathbf{x}-\mathbf{x}_{\mathbf{0}}\right) f \cos (\Omega t)$ applied at the point $\mathbf{x}_{\mathbf{0}}$ on the plate. The damping will take the form of modal viscous damping once the equations are discretised along the normal modes. The bilinear operator $L(\cdot, \cdot)$ is known as von Kármán operator [46] and, in Cartesian coordinates, it has the form of

$$
L(\alpha, \beta)=\alpha_{, x x} \beta_{, y y}+\alpha_{, y y} \beta_{, x x}-2 \alpha_{, x y} \beta_{, x y}
$$

where ${ }_{, s}$ denotes differentiation with respect to the variable $s$. This operator, although itself bilinear, is the source of the nonlinear terms in the equations. All the quantities are taken in their natural units, so that Eq. (1a) and Eq. (1b) have the dimensions, respectively, of $\mathrm{kg} \mathrm{m}^{-1} \mathrm{~s}^{-2}$ and $\mathrm{kg} \mathrm{m}^{-2} \mathrm{~s}^{-2}$. The term $L(w, w)$ in eq. (1b) is quadratic in $w$ and its derivatives, so once the solution for $F$ is injected into (1a), a cubic nonlinearity will appear, leading to a Duffing-type set of coupled ordinary differential equations (ODEs).

\subsection{Linear Modes}

The strategy adopted here to solve the von Kármán system makes use of the linear modes for the displacement $w$ and Airy stress function $F$. This strategy is particularly useful for investigating the free and forced vibrations of the system, in the sense that it allows for the reduction of the dynamics of the problem from an infinite number of degrees of freedom to a finite one. The eigenmodes for the displacement $w$ will be denoted by the symbol $\Phi_{k}(x, y)$ and thus $w(x, y, t)$ is written as

$$
w(x, y, t)=S_{w} \sum_{k=1}^{N_{w}} \frac{\Phi_{k}(x, y)}{\left\|\Phi_{k}\right\|} q_{k}(t),
$$

where $\Phi_{k}$ is such that

$$
\Delta \Delta \Phi_{k}(x, y)=\frac{\rho h}{D} \omega_{k}^{2} \Phi_{k}(x, y) .
$$


Note that the sum in eq. (3a) is terminated at $N_{w}$ in practice. The linear modes can be defined up to a constant of normalisation that can be chosen arbitrarily. For the sake of generality, $S_{w}$ here denotes the constant of normalisation of the function $\bar{\Phi}=S_{w} \frac{\Phi_{k}(x, y)}{\left\|\Phi_{k}\right\|}$. The norm is obtained from a scalar product $<\alpha, \beta>$ between two functions $\alpha(x, y)$ and $\beta(x, y)$, defined as

$$
<\alpha, \beta>=\int_{S} \alpha \beta d S \quad \longrightarrow \quad\left\|\Phi_{k}\right\|^{2}=<\Phi_{k}, \Phi_{k}>
$$

Eq. (3b) is the eigenvalue problem definition, and it is a Kirchhoff-like equation for linear plates.

The Airy stress function is expanded along an analogue series:

$$
\begin{aligned}
& F(x, y, t)=S_{F} \sum_{k=1}^{N_{F}} \frac{\Psi_{k}(x, y)}{\left\|\Psi_{k}\right\|} \eta_{k}(t), \\
& \Delta \Delta \Psi_{k}(x, y)=\zeta_{k}^{4} \Psi_{k}(x, y) .
\end{aligned}
$$

Boundary conditions for $w$ and $F$ will be specified in the next subsection. The linear modes so defined are orthogonal with respect to the scalar product, and are therefore a suitable function basis [25]. Orthogonality between two functions $\Lambda_{m}(x, y), \Lambda_{n}(x, y)$ is expressed as

$$
<\Lambda_{m}, \Lambda_{n}>=\delta_{m, n}\left\|\Lambda_{m}\right\|^{2}
$$

where $\delta_{m, n}$ is the Kroenecker delta.

Once the linear modal shapes are known, system (1) may then be reduced to a set of ordinary differential equations, each referring to the $k$-th modal coordinate $q_{k}(t), \quad k=1, \ldots, N_{w} . N_{w}$ represents the order of the system of ODEs.

\subsection{Reduction to a set of ODEs}

The introduction of the expansion series (3a) and (5a) allows for the decomposition of the original von Kármán problem onto a set of coupled, nonlinear ordinary differential equations (ODEs). As a starting point, eq. (5a) is substituted into eq. (1b) to obtain

$$
\eta_{k}=-\frac{E h}{2 \zeta_{k}^{4}} \frac{S_{w}^{2}}{S_{F}} \sum_{p, q} q_{p} q_{q} \frac{\int_{S} \Psi_{k} L\left(\Phi_{p}, \Phi_{q}\right) \mathrm{d} S}{\left\|\Psi_{k}\right\|\left\|\Phi_{p}\right\|\left\|\Phi_{q}\right\|}
$$

Integration is performed over the area of the plate, and the orthogonality relation is used. Injecting eq. (3) and (7) into eq. (1a) gives

$$
\begin{aligned}
\rho h S_{w} \sum_{k} \frac{\omega_{k}^{2} \Phi_{k}}{\left\|\Phi_{k}\right\|} q_{k}+\rho h S_{w} \sum_{k} \frac{\Phi_{k}}{\left\|\Phi_{k}\right\|} \ddot{q}_{k}+c S_{w} \sum_{k} \frac{\Phi_{k}}{\left\|\Phi_{k}\right\|} \dot{q}_{k} \\
\quad=-\frac{E h S_{w}^{3}}{2} \sum_{n, p, q, r} \frac{1}{\zeta_{n}^{4}} \frac{L\left(\Phi_{p}, \Psi_{n}\right)}{\left\|\Psi_{p}\right\|\left\|\Phi_{n}\right\|} \frac{\int_{S} \Psi_{n} L\left(\Phi_{q}, \Phi_{r}\right) \mathrm{d} S}{\left\|\Phi_{q}\right\|\left\|\Phi_{r}\right\|\left\|\Psi_{n}\right\|} q_{p} q_{q} q_{r}+\delta\left(\mathbf{x}-\mathbf{x}_{\mathbf{0}}\right) f \cos (\Omega t) .
\end{aligned}
$$

Then the equation is multiplied on both sides by $\Phi_{s}$ and integrated over the surface of the plate. The result is

$$
\ddot{q}_{s}+\omega_{s}^{2} q_{s}+2 \chi_{s} \omega_{s} \dot{q}_{s}=-\frac{E S_{w}^{2}}{2 \rho} \sum_{p, q, r}^{n} \frac{H_{q, r}^{n} E_{p, n}^{s}}{\zeta_{n}^{4}} q_{p} q_{q} q_{r}+\frac{\Phi_{s}\left(\mathbf{x}_{\mathbf{0}}\right)}{\left\|\Phi_{s}\right\| \rho h S_{w}} f \cos (\Omega t)
$$

where a modal viscous damping is introduced in the equation, scaled by $\chi_{s}=c /\left(2 \rho h \omega_{s}\right)$ (a dimensionless parameter). A practical advantage of the modal description is that $\chi_{s}$ can be estimated experimentally for a large number of modes [11] and so the modal approach allows the simulation of complex frequency dependent damping mechanisms with practically no extra effort.

Two third order tensors, $H_{q, r}^{n}$ and $E_{p, n}^{s}$ appear in eq. (9). These are defined as

$$
H_{p, q}^{n}=\frac{\int_{S} \Psi_{n} L\left(\Phi_{p}, \Phi_{q}\right) \mathrm{d} S}{\left\|\Psi_{n}\right\|\left\|\Phi_{p}\right\|\left\|\Phi_{q}\right\|}, \quad E_{r, n}^{s}=\frac{\int_{S} \Phi_{s} L\left(\Phi_{r}, \Psi_{n}\right) \mathrm{d} S}{\left\|\Phi_{r}\right\|\left\|\Phi_{s}\right\|\left\|\Psi_{n}\right\|} .
$$

It is seen that the ODEs are cubic with respect to the variables $q_{s}$, so a fourth order tensor $\Gamma$ can conveniently be introduced in the equations, as

$$
\Gamma_{p, q, r}^{s}=\sum_{n=1}^{N_{F}} \frac{H_{p, q}^{n} E_{r, n}^{s}}{2 \zeta_{n}^{4}} .
$$

Once the tensor $\Gamma$ is known, one is left with a set of coupled ODEs that can be integrated in the time variable using standard integration schemes. Alternatively, continuation methods can be employed to derive a complete bifurcation analysis of the nonlinear dynamics. 


\subsection{Boundary Conditions}

To recover the von Kármán equations, one may define the potential and kinetic energies of a bent plate, in the following way:

$$
\begin{aligned}
& V=\sum_{i, k=1}^{3} \frac{h}{2} \int_{S} \sigma_{i k} u_{i k} d S, \\
& T=\frac{\rho h}{2} \int_{S} \dot{w}^{2} d S, \\
& U=\sum_{i, k=1}^{2} \frac{h}{2} \int_{S} \tilde{\sigma}_{i k} \tilde{u}_{i k} d S,
\end{aligned}
$$

where $V, T$ are the potential and kinetic energies for pure bending, and $U$ is the potential energy for the stretching in the in-plane direction. Note that two strain tensors $\left(u_{i k}\right.$ and $\left.\tilde{u}_{i k}\right)$ and two stress tensors $\left(\sigma_{i k}\right.$ and $\left.\tilde{\sigma}_{i k}\right)$ are introduced, in order to account for the pure bending and in-plane energies; note also that the indices of the in-plane tensors can take only two values. Suppose that the displacement vector is $\mathbf{u}=\left(u_{x}, u_{y}, w\right)$ defined in a Cartesian set of coordinates $\mathbf{x}=(x, y, z)$. The symmetric strain tensor $u_{i k}$ is linear, and can be given in terms of the vertical displacement $w$ as follows [23]:

$$
u_{x x}=-z \partial^{2} w / \partial x^{2} ; \quad u_{y y}=-z \partial^{2} w / \partial y^{2} ; \quad u_{x y}=-z \partial^{2} w / \partial x \partial y ; \quad u_{z z}=\frac{v}{1-v} z\left(\partial^{2} w / \partial x^{2}+\partial^{2} w / \partial y^{2}\right)
$$

and zero for all the other components. The stress-strain relationships are also linear, as the material is assumed to be, and read

$$
\sigma_{i k}=\sum_{l=1}^{3} \frac{E}{1+v}\left(u_{i k}+\frac{v}{1-2 v} u_{l l} \delta_{i k}\right) .
$$

The symmetric, two-dimensional strain tensor $\tilde{u}_{i k}$ is nonlinear, and given by

$$
\tilde{u}_{i k}=\left[\frac{1}{2}\left(\frac{\partial u_{i}}{\partial x_{k}}+\frac{\partial u_{k}}{\partial x_{i}}\right)+\frac{1}{2} \frac{\partial w}{\partial x_{i}} \frac{\partial w}{\partial x_{k}}\right],
$$

and the stress-strain relationships for the in-plane stretching are given as

$$
\tilde{\sigma}_{x x}=\frac{E}{1-v^{2}}\left(\tilde{u}_{x x}+v \tilde{u}_{y y}\right) ; \quad \tilde{\sigma}_{y y}=\frac{E}{1-v^{2}}\left(\tilde{u}_{y y}+v \tilde{u}_{x x}\right) ; \quad \tilde{\sigma}_{x y}=\frac{E}{1+v} \tilde{u}_{x y} ;
$$

and zero for all the other components. The Airy stress function $F$ is introduced as

$$
\tilde{\sigma}_{x x}=\partial^{2} F / \partial y^{2} ; \quad \tilde{\sigma}_{y y}=\partial^{2} F / \partial x^{2} ; \quad \tilde{\sigma}_{x y}=-\partial^{2} F / \partial x \partial y
$$

Note that the only nonlinear term that appears in the definitions of the energies is the quadratic factor in $\tilde{u}_{i k}$. It is possible to make use of Hamilton's principle, stated in the form

$$
\int_{t_{0}}^{t_{1}} \delta(T-V-U) d t=0
$$

to recover the equations of motion (1) plus the boundary conditions. These can be categorised as follows [46] (here ${ }_{, n}, t$ denote differentiation along the normal and tangent directions respectively):

- In-plane direction

- free edge: $F_{, n t}=F_{, t t}=0$

- immovable edge $\left(w=0\right.$ along the boundary): $F_{, n n}-v F_{, t t}=F_{, n n n}+(2+v) F_{, n n t}=0$

- Edge rotation

- rotationally free: $w_{, n n}+v w_{, t t}=0$

- rotationally immovable $w_{, n}=0$

- Edge vertical translation

- free: $w_{, n n n}+(2-v) w_{, n t t}-\frac{1}{D}\left(F_{, t t} w_{, n}-F_{, n t} w_{t}\right)=0$

- immovable $w=0$ 
A corner condition arises as well, and it is

$$
w_{, x y}=0 \quad \text { at corners }
$$

This constraint has to be imposed as an extra condition only when the edge is transversely free. It is evident that the boundary conditions must be fulfilled by all the linear modes $\Phi_{k}, \Psi_{k}$ that appear in the expansions (3), (5). For the transverse function, simply supported boundary conditions are considered for the remainder of the paper. These describe a fixed, rotationally free edge and permit a simplified analysis because a solution is readily available:

$$
\Phi_{k}=\sin \left(\frac{k_{1} \pi x}{L_{x}}\right) \sin \left(\frac{k_{2} \pi y}{L_{y}}\right) ; \quad \omega_{k}^{2}=\frac{D}{\rho h}\left[\left(\frac{k_{1} \pi}{L_{x}}\right)^{2}+\left(\frac{k_{2} \pi}{L_{y}}\right)^{2}\right]^{2} .
$$

For the in-plane direction, a free edge is considered. However, a different form of the boundary conditions will be used, i.e. $F=F_{, n}=0$. It is evident that the assumed conditions are sufficient to satisfy the proper conditions $F_{, n t}=F_{, t t}=0$. Note that, mathematically speaking, the assumed conditions on $F$ turn the stress function problem into a transversely clamped plate problem.

The selected boundary conditions are also known as simply supported with movable edges [1].

\section{A solution for the clamped plate}

As shown in the previous section, the eigenvalue problem for $F$ with the chosen boundary conditions is equivalent to that of a clamped Kirchhoff plate. To this extent, the Galerkin method is employed, as an analytical solution for the problem is not available.

The starting point of the Galerkin method is to express the eigenfunction $\Psi_{k}$ of eq. (5) as a series of this form

$$
\Psi_{k}(x, y)=\sum_{n=0}^{N_{c}} a_{n}^{k} \Lambda_{n}(x, y)
$$

where $\Lambda_{n}(x, y)$ are the expansion functions depending on some index $n$, and $a_{n}^{k}$ are the expansion coefficients: these depend on the index $n$ and of course on the index $k$. The total number of expansion functions is $N_{c}$, and obviously the accuracy of the solution improves as this parameter is increased. The $\Lambda$ 's must be carefully selected from the set of all comparison functions [48]; this is to say that they need to satisfy the boundary conditions associated with the problem, that they are at least $p$ times differentiable (where $p$ is the order of the PDE), and they form a complete set over the domain of the problem. Completeness is quite a rather involved property to prove; however one generally resorts to variations of sine or cosine Fourier series, for which completeness follows directly from the Fourier theorem.

For this work, the expansion functions were selected according to a general method proposed in [30], where it is shown how a Kirchhoff plate problem can be solved by means of a double modified Fourier cosine series, i.e.

$$
\Lambda_{n}(x, y)=X_{n_{1}}(x) Y_{n_{2}}(y)=\left(\cos \left(\frac{n_{1} \pi x}{L_{x}}\right)+p_{n_{1}}(x)\right)\left(\cos \left(\frac{n_{2} \pi y}{L_{y}}\right)+p_{n_{2}}(y)\right),
$$

where $p_{n_{1}}(x), p_{n_{2}}(y)$ are fourth order polynomials in the variables $x$ and $y$, and depending as well on the integers $n_{1}, n_{2}$. Note that the order of the polynomials corresponds to the order of the PDE. The role of the polynomial is to account for possible discontinuities at the edges due to the boundary conditions. [30] is mainly concerned with a general solution strategy, where the plate is equipped with linear and rotational springs at the edges to simulate the effect of different boundary conditions. In [30] the polynomials of eq. (22) do not appear explicitly, as they are obtained through matrix inversion in order to comply with the general form of the boundary conditions. In turn, these matrices present the values of all the springs, and the general expression of the $\Lambda$ 's is rather involved. However, given that the focus here is on the clamped plate only, the analytical limit of all the springs having infinite stiffness is taken, so that an explicit form for (22) can indeed be recovered, and this is:

$$
X_{n_{1}}(x)=\cos \left(\frac{n_{1} \pi x}{L_{x}}\right)+\frac{15\left(1+(-1)^{n_{1}}\right)}{L_{x}^{4}} x^{4}-\frac{4\left(8+7(-1)^{n_{1}}\right)}{L_{x}^{3}} x^{3}+\frac{6\left(3+2(-1)^{n_{1}}\right)}{L_{x}^{2}} x^{2}-1,
$$

and similarly for $Y_{n_{2}}(y)$. Note that for the clamped plate, satisfaction of the boundary conditions is essential for a fast converging solution. This is because the conditions at the edges for the clamped plate are geometrical, as they prescribe the vanishing of the displacement and of the slope. Thus, an expansion function that does not satisfy these conditions could lead to slow converging solutions, if not to wrong results.

It is seen that this expansion satisfies the clamped plate conditions, but not the differential equation. It is possible to show however that one particular choice for the expansion coefficients $a_{n}^{k}$ will render the function $\Psi_{k}$ an eigenfunction for the problem. The Galerkin method describes how to build up stiffness and mass matrices in order to calculate the coefficient vector $a_{n}^{k}$ and the corresponding eigenfrequency $\zeta_{k}^{4}$. For the problem (5b), these matrices are

$$
K_{i j}=\int_{S}\left[\Delta \Lambda_{i} \Delta \Lambda_{j}-L\left(\Lambda_{i}, \Lambda_{j}\right)\right] d S, \quad \text { Stiffness Matrix }
$$



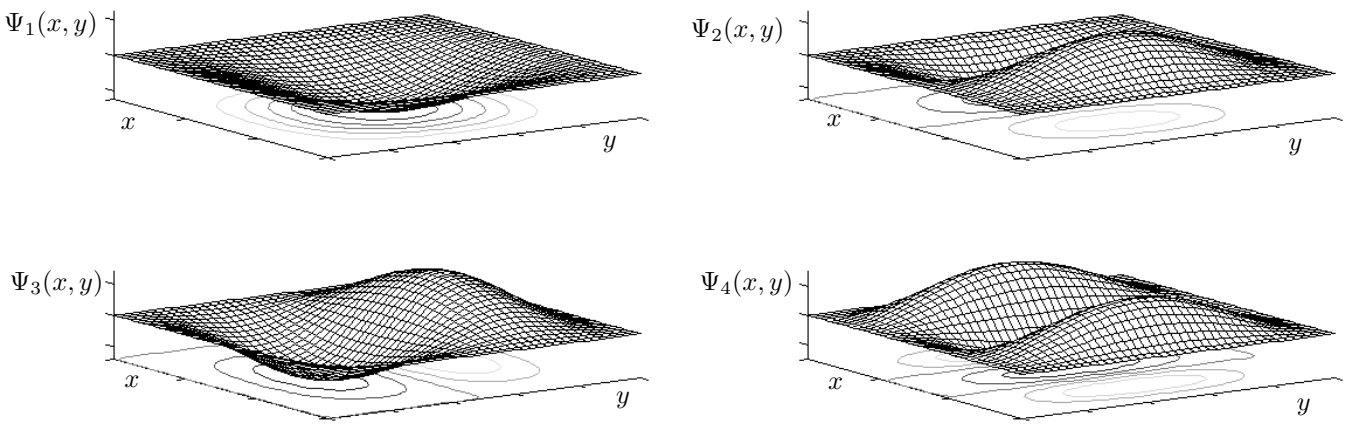

Fig. 1 First four modes for the clamped plate, $\xi=2 / 3$.

$$
M_{i j}=\int_{S} \Lambda_{i} \Lambda_{j} d S, \quad \text { Mass Matrix }
$$

where $L(\cdot, \cdot)$ is the von Kármán operator. Note that the integrals can be calculated analytically, because of the simple form of the expansion function. Explicit forms of the integrals are presented in appendix A. Then

$$
K \mathbf{a}=\zeta^{4} M \mathbf{a}
$$

which is the required eigenvalue problem that leads to the expansion coefficients and the eigenvalues.

\subsection{Numerical Results for the Clamped Plate}

In this section, the results obtained by Galerkin's method are compared to the classical results found in Leissa's tables [29]. A finite difference scheme (FD) developed by Bilbao [5] is as well used as a benchmark. A useful parameter in plate problems is the aspect ratio, here defined as $L x / L y$ and denoted by the symbol $\xi$. Assume that two plates present the same aspect ratio: then it is straightforward to show that the quantity $\zeta^{2} L_{x} L_{y}$ is constant for the two plates, where $\zeta$ is defined in eq. (5b) (thus making $\zeta^{2} L_{x} L_{y}$ a nondimensional parameter). As a first step, the rate of convergence of the eigenfrequencies is proposed in table 1 . The plate has an aspect ratio of $2 / 3 . N_{c}$ denotes the number of modes kept in the expansion (21). Note that convergence for the first 100 eigenfrequencies is obtained up to the fifth significant digit when

Table 1 Convergence of clamped plate frequencies, $\zeta_{k}^{2} L_{x} L_{y}, \xi=2 / 3$

$N_{c}$

\begin{tabular}{ccccccc}
$\mathbf{k}$ & 25 & 100 & 144 & 255 & 400 & 484 \\
\cline { 2 - 6 } $\mathbf{1}$ & 40.509 & 40.508 & 40.508 & 40.508 & 40.508 & 40.508 \\
$\mathbf{2}$ & 62.563 & 62.556 & 62.556 & 62.556 & 62.556 & 62.556 \\
$\mathbf{3}$ & 99.193 & 99.187 & 99.187 & 99.186 & 99.186 & 99.186 \\
$\mathbf{4}$ & 99.790 & 99.787 & 99.783 & 99.783 & 99.783 & 99.783 \\
$\mathbf{5}$ & 119.75 & 119.71 & 119.71 & 119.71 & 119.71 & 119.71 \\
$\mathbf{2 0}$ & 476.05 & 359.60 & 359.58 & 359.57 & 359.57 & 359.57 \\
$\mathbf{5 0}$ & - & 859.52 & 839.38 & 839.31 & 839.31 & 839.31 \\
$\mathbf{1 0 0}$ & - & 2439.9 & 1669.7 & 1574.3 & 1500.3 & 1500.3
\end{tabular}

$N_{c}=400$. This corresponds to a calculation time of less than 10 seconds in MATLAB on a standard machine equipped with an Intel Core i5 CPU 650 @ 3.20GHz, and a memory of 4GB. In table 2, the results obtained by Galerkin's method are compared to those found in Leissa and as well as to the outcome of the FD scheme. For this, the plate parameters have been set as: $L_{x}=0.4 \mathrm{~m}, L_{y}=0.6 \mathrm{~m}, \rho=7860 \mathrm{~kg} / \mathrm{m}^{3}, v=0.3, h=0.001 \mathrm{~m}, E=2 \cdot 10^{11} \mathrm{~Pa}$. The FD scheme employs $241 \times 161$ discretisation points, so that $\frac{\Delta x \Delta y}{S}=2.6 \cdot 10^{-5}$. Even though Leissa's book represents one of the main references in the area of plate eigenmodes and frequencies, its results are somehow outdated, being about 40 years old. Thus, discrepancies between the presented Galerkin's method and the numbers from Leissa's book are not at all concerning. On the other hand, it is known that FD schemes converge at a slower rate than a pure modal approach. This is a consequence of the fact that FD schemes rely on discrete grid meshes. Convergence for the first eigenfrequencies for the plate using the FD scheme is presented in table 3. Note that the eigenfrequencies tend to converge to the same values as the Galerkin's method. However, the calculation time in MATLAB for a mesh grid of $280 \times 419$ points is 
Table 2 Comparison of clamped plate frequencies, $\zeta_{k}^{2} L_{x} L_{y}, \xi=2 / 3$

\begin{tabular}{cccc} 
& & Source & \\
$\mathbf{k}$ & $\begin{array}{c}\text { Galerkin } \\
\left(N_{c}=400\right)\end{array}$ & Leissa & FD $(241 \times 161)$ \\
\cline { 2 - 4 } $\mathbf{1}$ & 40.51 & 40.51 & 40.05 \\
$\mathbf{2}$ & 62.56 & 62.58 & 61.93 \\
$\mathbf{3}$ & 99.19 & 98.25 & 98.00 \\
$\mathbf{1 0}$ & 208.0 & 207.9 & 205.5 \\
$\mathbf{2 0}$ & 359.6 & - & 355.32
\end{tabular}

Table 3 Convergence of clamped plate frequencies, FD scheme, $\zeta_{k}^{2} L_{x} L_{y}, \xi=2 / 3$

\begin{tabular}{ccccccc} 
& \multicolumn{5}{c}{ Grid Points } \\
$\mathbf{k}$ & $36 \times 54$ & $51 \times 76$ & $114 \times 171$ & $161 \times 241$ & $228 \times 342$ & $280 \times 419$ \\
\cline { 2 - 7 } $\mathbf{1}$ & 38.539 & 39.094 & 39.862 & 40.048 & 40.182 & 62.115 \\
$\mathbf{2}$ & 59.889 & 60.638 & 61.682 & 61.934 & 98.343 & 62.196 \\
$\mathbf{4}$ & 93.993 & 95.484 & 97.509 & 97.995 & 99.134 & 98.499 \\
$\mathbf{1 0}$ & 95.768 & 96.914 & 98.491 & 98.865 & 206.22 & 206.253
\end{tabular}

Table 4 Convergence of clamped plate frequencies, $\zeta_{k}^{2} L_{x} L_{y}, \xi=1$ (square plate)

$\begin{array}{ccccccc}\mathbf{k} & 25 & 100 & 144 & 255 & 400 & 384 \\ \mathbf{1} & 35.986 & 35.985 & 35.985 & 35.985 & 35.985 & 73.394 \\ \mathbf{2} & 73.398 & 73.394 & 73.394 & 73.394 & 73.394 & 73.394 \\ \mathbf{3} & 73.398 & 73.394 & 73.394 & 73.394 & 108.22 & 13.394 \\ \mathbf{4} & 108.24 & 108.22 & 108.22 & 108.22 & 131.58 & 131.58 \\ \mathbf{5} & 131.60 & 131.58 & 131.58 & 371.35 & 371.34 & 371.34 \\ \mathbf{2 0} & 376.42 & 371.37 & 371.35 & 805.35 & 805.34 & 805.34 \\ \mathbf{5 0} & - & 805.89 & 805.42 & 1546.2 & 1546.1 & 1546.1\end{array}$

much slower (about 20 minutes). Table 4 presents the eigenfrequencies for the square plate, using Galerkin's method. It is possible to appreciate the same rate of convergence as for the previous case. Again, the results are compared with Leissa and to the FD scheme outcome $(161 \times 161$ grid points $)$ in table 5. Plots of some clamped plate eigenmodes are presented in figure 1. These results show that the Galerkin method, with the carefully chosen expansion (23), is indeed

Table 5 Comparison of clamped plate frequencies, $\zeta_{k}^{2} L_{x} L_{y}, \xi=1$ (square plate)

\begin{tabular}{cccc} 
& & \multicolumn{3}{c}{ Source } \\
$\mathbf{k}$ & $\begin{array}{c}\text { Galerkin } \\
\left(N_{c}=400\right)\end{array}$ & Leissa & FD $(161 \times 161)$ \\
\cline { 2 - 4 } $\mathbf{1}$ & 35.98 & 35.99 & 35.54 \\
$\mathbf{2}$ & 73.39 & 73.41 & 72.49 \\
$\mathbf{3}$ & 73.39 & 73.41 & 72.49 \\
$\mathbf{4}$ & 108.2 & 108.3 & 106.9 \\
$\mathbf{2 0}$ & 371.3 & - & 366.7
\end{tabular}

a fast converging strategy for the calculation of the eigenfrequencies, as it allows for precisely computing hundreds of modes within seconds.

\section{The nonlinear coupling coefficients}

\subsection{Symmetry Properties}

In this section symmetry properties for the coupling coefficients $\Gamma$ that appear in eq. (11) are presented. First, it is obvious that

$$
H_{p, q}^{i}=H_{q, p}^{i},
$$


because of the symmetry of the operator $L(\cdot, \cdot)$. Secondly, integrating by parts the integral in the definition of $E$ in eq. (10) gives

$$
\begin{aligned}
\left\|\Psi_{q}\right\|\left\|\Phi_{n}\right\|\left\|\Phi_{p}\right\| E_{p, q}^{n} & =\oint\left[\Phi_{n} \Psi_{q, y} \Phi_{p, x x}-2 \Phi_{n} \Psi_{q, x} \Phi_{p, x y}-\Psi_{q} \frac{\partial}{\partial y}\left(\Phi_{n} \Phi_{p, x x}\right)\right] \mathbf{y} \cdot \mathbf{n} d \Omega+ \\
& +\oint\left[\Phi_{n} \Psi_{q, x} \Phi_{p, y y}+2 \Psi_{q} \frac{\partial}{\partial y}\left(\Phi_{n} \Phi_{p, x y}\right)-\Psi_{q} \frac{\partial}{\partial x}\left(\Phi_{n} \Phi_{p, y y}\right)\right] \mathbf{x} \cdot \mathbf{n} d \Omega+\int \Psi_{q} L\left(\Phi_{p}, \Phi_{n}\right) d S .
\end{aligned}
$$

It is easy to see that the selected boundary conditions make the surface integrals vanish, so that the following property holds

$$
E_{p, q}^{n}=H_{p, n}^{q}
$$

In this way, the tensor $\Gamma$ may then be conveniently written as

$$
\Gamma_{p, q, r}^{s}=\sum_{n=1}^{N_{F}} \frac{H_{p, q}^{n} H_{r, s}^{n}}{2 \zeta_{n}^{4}} .
$$

Note that the tensor $H$ as defined in eq. (10) is divided by the norms of the modes, so the value of $\Gamma$ is independent of the particular choice for the constants $S_{w}, S_{F}$ in eqs. (3b), (5b). Basically the symmetry properties for $\Gamma$ mean the following sets of indices will produce the same numerical value:

$$
(s, p, q, r),(r, p, q, s),(s, q, p, r),(r, q, p, s),(q, r, s, p),(p, r, s, q),(q, s, r, p),(p, s, r, q)
$$

These symmetry properties can lead to large memory savings when the number of transverse and in-plane modes is a few hundred.

\subsection{Null Coupling Coefficients}

For the sake of numerical computation, it would be interesting to know a priori which coupling coefficients are null. In actual fact, empirical observations of the $\Gamma$ tensor suggest that only a smaller fraction of coefficients is not zero. As an example, consider table 6 where the nonzero values for the coefficients $\Gamma_{5, q, r}^{1}$ for a plate with $\xi=2 / 3$ were collected (with $p, q=1 \ldots 10$ ): the table presents only 24 nonzero coefficients out of a total of 100. These coefficients measure the amount of interaction between the different transverse modes. As a matter of fact, the modes can be classified according to the symmetry with respect to the $x$ and $y$ axis where the origin is placed at the centre of the plate. Four families exist, and these are: doubly symmetric (SS), antisymmetric-symmetric (AS and SA) and doubly antisymmetric (AA). For instance, the first mode is a doubly-symmetric mode because it presents one maximum at the centre of the plate, and is thus symmetric with respect to the two orthogonal directions departing from the centre of the plate in the $x$ and $y$ directions, whereas mode 5 is AA. The first sixteen modes for the case under study may be classified in the following groups:

$$
\begin{array}{ll}
\circ & \text { SS: } 1,4,8,11,12 \\
\circ & \text { SA: } 2,7,9,14,16 \\
\circ & \text { AS: } 3,6,13,15 \\
\circ & \text { AA: } 5,10
\end{array}
$$

This list will become useful when interpreting the free vibration diagrams of the next section. Remarkably, the number of indices of the $\Gamma$ coefficients (four) matches the number of modal shape sets. Table 6 presents the modal families to which the interacting modes belong; observation of alike tables permits to state the following heuristic rule:

the indices $(s, p, q, r)$ will give a nonzero value for $\Gamma_{p, q, r}^{s}$ if and only if modes s,p,q,r come all from distinct modal shape groups or if they come from the same group two by two.

For example, the combinations (SS, SS, AS, SA) and (SS, SS, SS, AS) will definitely give a zero value; on the other hand the combinations (SS, SS, SS, SS), (SS, AA, SS, AA) and (SS, AS, SA, AA) will give a nonzero value. A rigorous mathematical proof is not carried out as it involves a rather lengthy development which is beyond the scope of the present work. However, it has been numerically checked for a large number of $\Gamma$ 's involving a few hundred modes, providing an exhaustive verification of this rule.

This rule, in combination with the previous remarks on symmetry, can be used to speed up the calculation of the $\Gamma$ tensor (for example by pre-allocating the zero entries when using a sparse matrix description). In some way, this observation relates to the already noted property of von Kármán shells [47]. There, the coupling rules are actually more involved, but they can be somehow more directly proved mathematically. 
Table 6 Nonzero $\Gamma_{5, q, r}^{1}\left(L_{x} L_{y}\right)^{3}, \xi=2 / 3$, for $q=1: 10, r=1: 10$

\begin{tabular}{rrccrrrc}
\hline value & $q$ & $r$ & Modal Shape Groups & value & $q$ & $r$ & Modal Shape Groups \\
\hline 21.36 & 1 & 5 & SS AA SS AA & 27.55 & 6 & 2 & SS AA AS SA \\
-21.75 & 1 & 10 & SS AA SS AA & 150.98 & 6 & 7 & SS AA AS SA \\
48.46 & 2 & 3 & SS AA SA AS & 36.52 & 6 & 9 & SS AA AS SA \\
7.55 & 2 & 6 & SS AA SA AS & -72.47 & 7 & 3 & SS AA SA AS \\
122.11 & 3 & 2 & SS AA AS SA & 119.51 & 7 & 6 & SS AA SA AS \\
-169.47 & 3 & 7 & SS AA AS SA & 56.36 & 8 & 5 & SS AA SS AA \\
-69.44 & 3 & 9 & SS AA AS SA & -64.89 & 8 & 10 & SS AA SS AA \\
56.71 & 4 & 5 & SS AA SS AA & 10.19 & 9 & 3 & SS AA SA AS \\
9.8 & 4 & 10 & SS AA SS AA & 65.63 & 9 & 6 & SS AA SA AS \\
3.1 & 5 & 1 & SS AA AA SS & -51.96 & 10 & 1 & SS AA AA SS \\
144.68 & 5 & 4 & SS AA AA SS & 97.76 & 10 & 4 & SS AA AA SS
\end{tabular}

\subsection{A few words on the FD scheme}

To validate the computational results for the $\Gamma$ tensor, an FD scheme developed in [5] has been extensively used. In this sense, the role of the discretised $L$ operator in eq. (11) is central. For two discrete functions $\alpha, \beta$ defined over the plate grid, the form for the discrete counterpart $l(\alpha, \beta)$ has been selected as

$$
l(\alpha, \beta)=\delta_{x x} \alpha \delta_{y y} \beta+\delta_{y y} \alpha \delta_{x x} \beta-2 \mu_{x-} \mu_{y-}\left(\delta_{x+y+} \alpha \delta_{x+y+} \beta\right) .
$$

The $\delta$ 's are discrete derivative operators and the $\mu$ 's are averaging operators, as follows

$$
\delta_{x x}=\frac{1}{h_{x}^{2}}\left(e_{x+}-2+e_{x-}\right) ; \quad \delta_{x+}=\frac{1}{h_{x}}\left(e_{x+}-1\right) ; \mu_{x-}=\frac{1}{2}\left(e_{x-}+1\right),
$$

where $e_{x+}\left(e_{x-}\right)$ is the positive (negative) shifting operator and $h_{x}$ is the step size along the $x$ direction. Note that this particular choice for the $l$ operator is due to the fact that it produces an energy-conserving scheme, as explained exhaustively in [5]. The eigenmodes are obtained by solving discrete counterparts of eqs. (3b) and (5b), thus a discrete double Laplacian is needed. At interior points, it can be approximated by

$$
\delta_{\Delta \boxplus} \delta_{\Delta \boxplus}=\left(\delta_{x x}+\delta_{y y}\right)\left(\delta_{x x}+\delta_{y y}\right)=\Delta \Delta+O\left(h_{x} h_{y}\right) .
$$

Enforcing of boundary conditions (simply supported and clamped) is described in [6]. Once the modes are known, one makes use of (31) to get the values of the coupling coefficients in eq. (11).

\subsection{Numerical Results}

In this subsection some numerical results are presented. It is somehow useful to note that the $\Gamma$ 's depend only on the aspect ratio. In other words the quantity

$$
\Gamma_{p, q, r}^{s}\left(L_{x} L_{y}\right)^{3}
$$

is constant for all the plates sharing the same aspect ratio. Table 7 presents a convergence test for a plate of aspect ratio $\xi=2 / 3$. The convergence in this case depends on two factors: the first is the amount of stress function modes retained in the definition of $\Gamma\left(N_{F}\right.$ in eq. (11)); the second is the accuracy on the Airy stress function modes and frequencies (quantified by the number $N_{c}$ in eq. (21)). For clarity, in the following tables $N_{F}$ is always the same as $N_{c}$. It is seen that a four-digit convergence up to the $\Gamma_{100,100,100}^{100}$ coefficient is obtained when $N_{F}=484$, and thus the convergence rate for these coefficients is slower than that of the stress functions eigenfrequencies alone. For the FD scheme, convergence

Table 7 Convergence of coupling coefficients, $\Gamma_{k, k, k}^{k}\left(L_{x} L_{y}\right)^{3}, \xi=2 / 3$

\begin{tabular}{ccccccc} 
& \multicolumn{3}{c}{$N_{F}$} \\
$\mathbf{k}$ & 100 & 144 & 225 & 400 & 484 & 625 \\
$\mathbf{n}$ & 20.033 & 20.034 & 20.034 & 20.034 & 20.034 & 20.034 \\
$\mathbf{2 0}$ & $7.5605 \cdot 10^{3}$ & $9.4893 \cdot 10^{3}$ & $9.4960 \cdot 10^{3}$ & $9.4970 \cdot 10^{3}$ & $9.4975 \cdot 10^{3}$ & $9.4977 \cdot 10^{3}$ \\
$\mathbf{5 0}$ & $1.3928 \cdot 10^{4}$ & $1.3929 \cdot 10^{4}$ & $1.3937 \cdot 10^{4}$ & $1.3937 \cdot 10^{4}$ & $1.3937 \cdot 10^{4}$ & $1.3937 \cdot 10^{4}$ \\
$\mathbf{1 0 0}$ & $1.4847 \cdot 10^{4}$ & $2.7360 \cdot 10^{4}$ & $1.2413 \cdot 10^{5}$ & $1.3334 \cdot 10^{5}$ & $2.2100 \cdot 10^{5}$ & $2.2108 \cdot 10^{5}$
\end{tabular}


and $N_{F}=200$, respectively. Note that, contrary to what whappens for the eigenfrequencies, convergence for the coupling coefficients is from above for FD and from below for the modal approach. It is also evident that a sufficiently large number of stress modes has to be retained to calculate reasonable approximate values for the $\Gamma$ 's: failing to do so may result in completely erroneous estimates (see for instance the last row of table 8 compared to the last row of table 9).

Table 8 Convergence of coupling coefficients, FD scheme, $\Gamma_{k, k, k}^{k}\left(L_{x} L_{y}\right)^{3}, \xi=2 / 3, N_{F}=100$

Grid Points

\begin{tabular}{ccccccc}
$\mathbf{k}$ & $36 \times 54$ & $51 \times 76$ & $114 \times 171$ & $161 \times 241$ & $228 \times 342$ & $280 \times 419$ \\
\cline { 2 - 7 } $\mathbf{1}$ & 21.113 & 20.523 & 20.523 & 20.380 & 20.252 & 20.188 \\
$\mathbf{2 0}$ & $9.8904 \cdot 10^{3}$ & $9.7238 \cdot 10^{3}$ & $9.6364 \cdot 10^{3}$ & $9.5761 \cdot 10^{3}$ & $9.5218 \cdot 10^{3}$ & $9.4944 \cdot 10^{3}$ \\
$\mathbf{5 0}$ & $1.4542 \cdot 10^{4}$ & $1.4430 \cdot 10^{4}$ & $1.4319 \cdot 10^{4}$ & $1.4224 \cdot 10^{4}$ & $1.4124 \cdot 10^{4}$ & $1.4070 \cdot 10^{4}$ \\
$\mathbf{1 0 0}$ & $1.0864 \cdot 10^{4}$ & $8.2016 \cdot 10^{3}$ & $6.8281 \cdot 10^{3}$ & $5.9133 \cdot 10^{3}$ & $5.1224 \cdot 10^{3}$ & $4.7387 \cdot 10^{3}$
\end{tabular}

Table 9 Convergence of coupling coefficients, FD scheme, $\Gamma_{k, k, k}^{k}\left(L_{x} L_{y}\right)^{3}, \xi=2 / 3, N_{F}=225$

Grid Points

\begin{tabular}{cccccccc}
$\mathbf{k}$ & $36 \times 54$ & $51 \times 76$ & $114 \times 171$ & $161 \times 241$ & $228 \times 342$ & $280 \times 419$ \\
\cline { 2 - 7 } $\mathbf{1}$ & 21.114 & 20.728 & 20.523 & 20.381 & 20.253 & 9.189 \\
$\mathbf{2 0}$ & $9.9634 \cdot 10^{3}$ & $9.7935 \cdot 10^{3}$ & $9.7035 \cdot 10^{3}$ & $9.6413 \cdot 10^{3}$ & $9.5851 \cdot 10^{3}$ & $9.5567 \cdot 10^{3}$ \\
$\mathbf{5 0}$ & $1.4552 \cdot 10^{4}$ & $1.4440 \cdot 10^{4}$ & $1.4329 \cdot 10^{4}$ & $1.4234 \cdot 10^{4}$ & $1.4134 \cdot 10^{4}$ & $1.4080 \cdot 10^{4}$ \\
$\mathbf{1 0 0}$ & $2.0268 \cdot 10^{5}$ & $2.0223 \cdot 10^{5}$ & $2.0227 \cdot 10^{5}$ & $2.0246 \cdot 10^{5}$ & $2.0271 \cdot 10^{5}$ & $2.0286 \cdot 10^{5}$
\end{tabular}




\section{Analysis of the periodic solutions}

The nonlinear dynamics of the plate is now analysed in terms of periodic solutions. The periodic orbits of the conservative system, also called the nonlinear normal modes (NNMs) [53] are first computed thanks to a pseudo arc-length numerical continuation method implemented in the software AUTO [19]. The amplitude-frequency relationships (i.e. the backbone curves) are exhibited for the first two modes up to 3-4 times the thickness, displaying a complicated network of bifurcation branches generated by internal resonances and modal couplings. Secondly, the forced responses of the damped plate are computed and their relationship with the backbone curve illustrated.

\subsection{Mode 1}

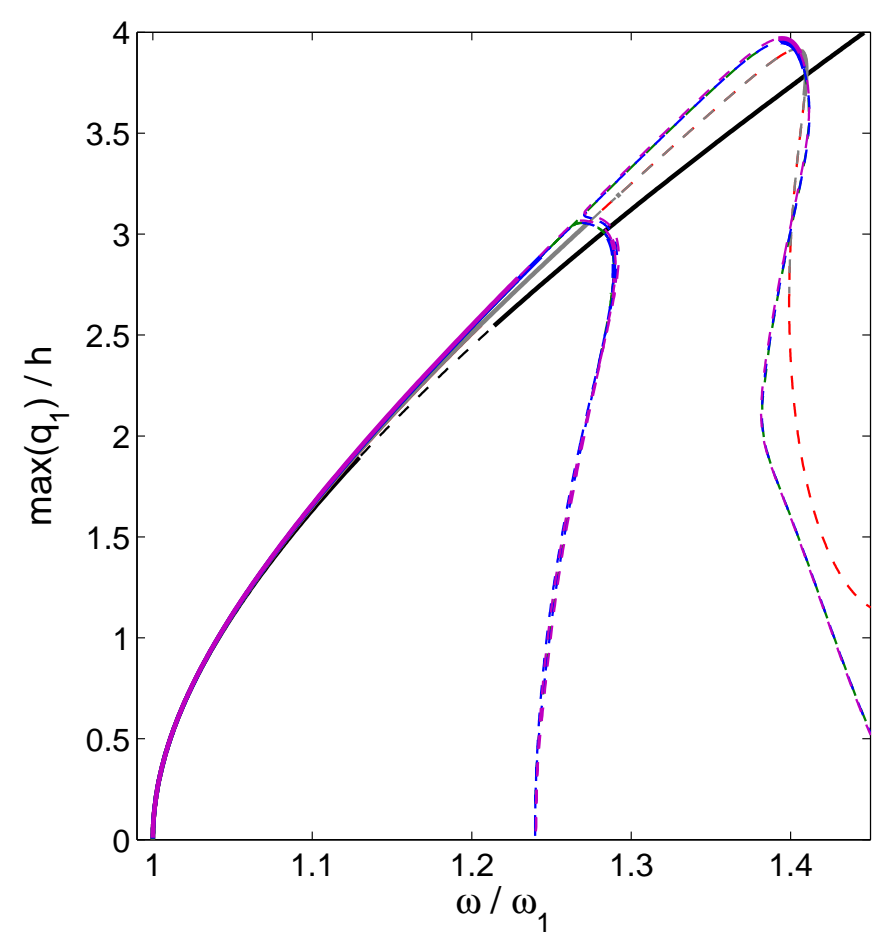

Fig. 2 Backbone curve (principal branch) convergence for mode 1: $N_{w}=6$ (black), $N_{w}=8$ (red), $N_{w}=10$ (grey), $N_{w}=14$ (green), $N_{w}=16$ (blue), $N_{w}=18$ (purple).

\subsubsection{Free Vibrations}

Fig. 2 is an illustration of the backbone convergence, for mode 1. The backbone is the curve obtained by plotting the maxima of the periodic solutions, in the case of undamped, unforced vibrations, which can be stable (continuous lines) or unstable (dashed lines). Note that only the principal branch is represented, thus the figure does not take into account the secondary branches departing from the bifurcation points. The figure presents the six backbones obtained when $N_{w}=6,8,10,14,16,18$. It is evident that the period of the vibration decreases as the amplitude increases, thus the curves bend to the right in the diagram; this behaviour is known in the literature as hardening-type nonlinearity. The backbone curves obtained for $N_{w}=14,16,18$ are almost exactly superimposed showing the convergence of the main solution branch for vibration amplitudes up to $4 h$. Note also that the cases $N_{w}=8,10$ are exactly superimposed because modes 9 and 10 do not belong to SS (the family of mode 1); hence the shape of the backbone does not change, although the stability intervals do not coincide. No stable solutions are detected by AUTO for vibrations larger than $4 h$ : this result is consistent with numerous experimental and numerical simulations of large amplitude vibrations of plates; higher vibration amplitudes give way to unstable solutions, in quasiperiodic or turbulent regimes [49,50]. The range of convergence of the backbone decreases when less modes $N_{w}$ are considered; particularly for the case of $N_{w}=6$ the backbone displays significant differences from the converged solution. In addition unstable solutions in this case set in much earlier, leading to the conclusion that when $N_{w}=6$ the backbone curve depicts an unrealistic scenario for amplitudes larger than $1.8 \mathrm{~h}$. The principal branch for the cases $N_{w}=14, N_{w}=16, N_{w}=18$ undergo an internal resonance around $\omega / \omega_{1} \approx 1.27$. This is a resonance between mode 1 and mode 11 , and will be commented later. It is seen that 
the cases $N_{w}=16, N_{w}=18$ are perfectly superimposed, thus a total number of $N_{w}=16$ modes is sufficient for full convergence; hence this is the number of modes that will be considered in the remainder of the paper. Fig. 3 shows the complete resonance scenario for mode 1 , in other words it presents the backbone and the bifurcated branches. Fig. 3 is basically a representation of the first NNM as a function of the frequency of vibration for the first mode. For clarity, only the most significant modal coordinates are represented. Branches are denoted by the symbol $\mathbf{B}_{k}^{i}$ where the index $i$ refers to the branch number and $k$ is the coordinate involved. Thus $\mathbf{B}^{1}$ is the main (backbone) branch, and $\mathbf{B}^{2}, \mathbf{B}^{3}, \ldots$ are secondary branches featuring a sudden loss of energy of $q_{1}$ in favour of other nonlinearly resonant modes. The appearance of internal resonance tongues due to the exchange of energy between modes at nonlinear frequencies of vibration has been previously observed for systems involving a few degrees of freedom, or for continuous systems with local nonlinearities $[8,32,26,41]$; in turn, these works show that NNM branches may fold in the presence of internal resonances. In this paper internal resonance foldings in the NNM branches are reported for a continuous structure with distributed geometric nonlinearity. The bifurcated branches are composed mainly by unstable states along intricate paths, and are difficult to compute numerically when using continuation. Note however that the free NNM is a physical abstraction: when damping and forcing are introduced in the system, most of the complicated details disappear, as it will be shown in the next subsection.

Observing $\mathbf{B}^{1}$ before the first bifurcation point, it is easily seen that modes 4 ( $\mathbf{B}_{\mathbf{4}}^{\mathbf{1}}$, green), 8 ( $\mathbf{B}_{\mathbf{8}}^{\mathbf{1}}$, light green), 11 $\left(\mathbf{B}_{\mathbf{1 1}}^{\mathbf{1}}\right.$, magenta) and 12 (not shown) bear a relatively important contribution. Here a typical nonresonant coupling is at hand. As it can be deduced from section 4.2, the only non-vanishing coefficients $\Gamma_{1,1,1}^{p}$ with $p=1, \ldots, 16$ are obtained for $p=1,4,8,11,12$. These coefficients are of prime importance as they give rise to a term of the form $\Gamma_{1,1,1}^{p} q_{1}^{3}$ in the equation for $q_{p}$. Thus when $q_{1}$ is large, modes $4,8,11,12$ acquire nonnegligible energy through the nonresonant coupling terms $\Gamma_{1,1,1}^{p}$ which act on the modal equations as forcing terms. These coefficients have been referred to as invariantbreaking terms because they have the property of breaking the invariance of the linear normal modes through modal coupling $[51,52]$. The coupling in these cases is nonresonant because no commensurability relationship exists between the frequencies of vibration.
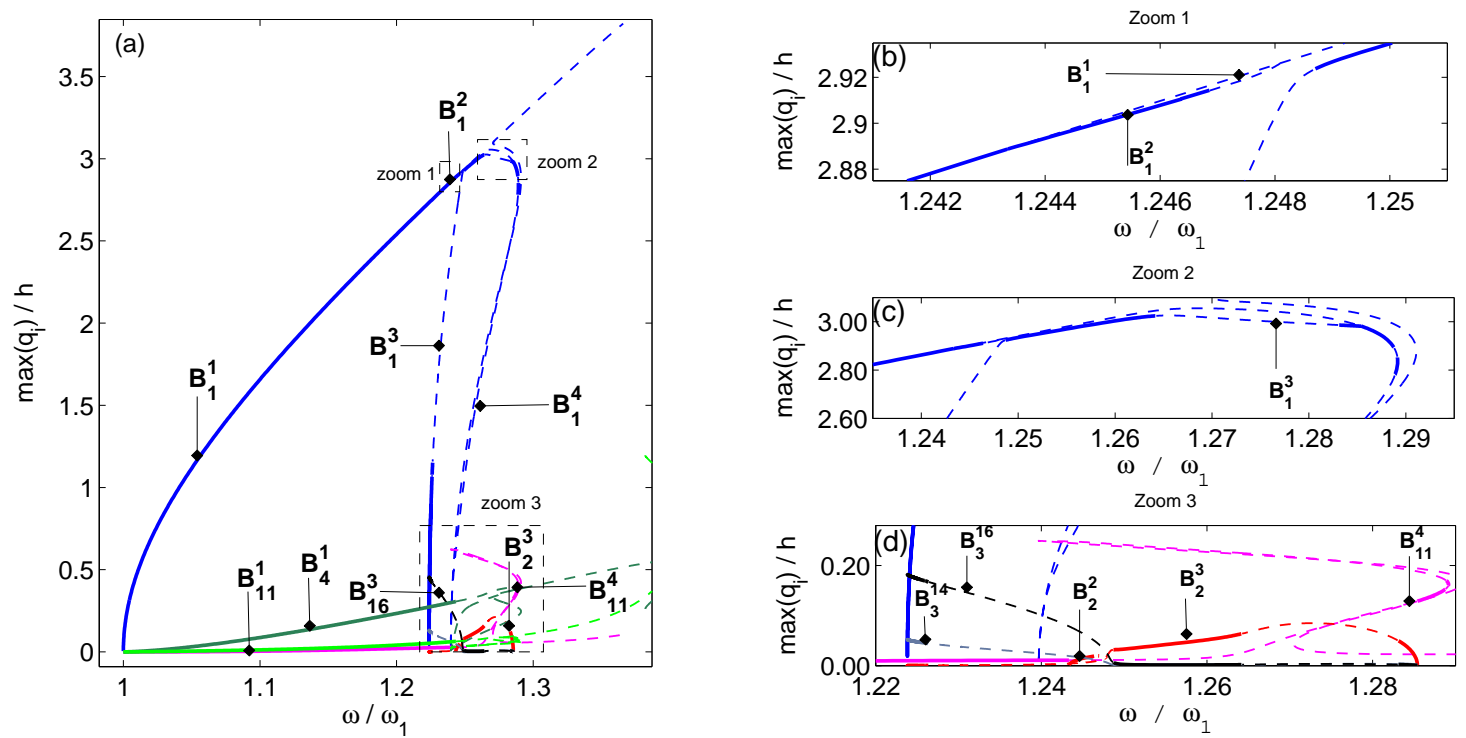

Fig. 3 (a): Free vibration diagram for mode 1, $N_{w}=16$. (b), (c), (d): Bifurcated branches and internal resonances.

The first bifurcated branch is $\mathbf{B}^{2}$ and develops along a very narrow frequency interval between $1.2435<\omega / \omega_{1}<$ 1.248. It is a very small branch and it is visible in fig.3(b) $\left(\mathbf{B}_{\mathbf{1}}^{\mathbf{2}}\right)$ and fig.3(d) $\left(\mathbf{B}_{\mathbf{2}}^{\mathbf{2}}\right)$. The modes involved in this bifurcation are 1 and 2. It is evident that mode 2, so far quiescent, is activated by an internal resonance with mode 1 . The order of the internal resonance can be obtained from a temporal simulation of the system comprising $N_{w}=16$ modes, fed at the input by the maximum displacements and velocities for all the modal coordinates along $\mathbf{B}^{\mathbf{2}}$. In this work, a fourth-order Runge-Kutta scheme is used for the time integration, giving at the output the oscillation in time for all the modes in the periodic regime. Fig. 4(a) represents modes 1 and 2 in the time domain on the point at $\omega / \omega_{1}=1.246$ along the branch $\mathbf{B}^{2}$. The figure shows that the period of vibration for mode 2 is exactly half the period of mode 1 , resulting in a 1:2 internal resonance. Note that starting the simulation on any other point of the same branch will lead to the same resonance ratio.

In the next section it will be seen that the bifurcation giving rise to $\mathbf{B}^{2}$ is key to the dynamics of the driven damped oscillations: this branch tends to occupy larger portions of the phase space as the forcing and damping terms increase, modifying the local structure of the invariant NNM manifold.

Following the principal branch in fig. 3(b) one encounters a second bifurcation giving rise to $\mathbf{B}^{\mathbf{3}}$. This is an interesting branch where again quiescent modes are activated by internal resonances. Fig. 3(d) reveals that these are modes $2\left(\mathbf{B}_{2}^{\mathbf{3}}\right.$, 

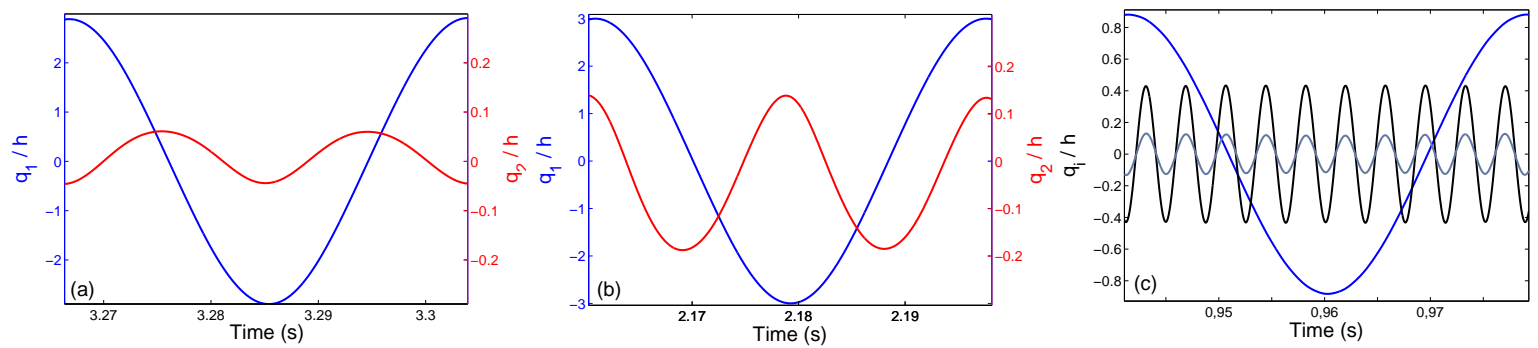

Fig. 4 (a): Modes 1 (blue) and 2 (red) along $\mathbf{B}^{\mathbf{2}}$ displaying 1:2 internal resonance. (b): Modes 1 (blue) and 2 (red) along $\mathbf{B}^{\mathbf{3}}$ displaying 1:2 internal resonance. (c): Modes 1 (blue), 14 (grey) and 16 (black) along $\mathbf{B}^{\mathbf{3}}$ displaying 1:10 internal resonance.

red), $14\left(\mathbf{B}_{\mathbf{1 4}}^{\mathbf{3}}\right.$, grey) and 16 ( $\mathbf{B}_{\mathbf{1 6}}^{\mathbf{3}}$, black). Note that the branch $\mathbf{B}^{\mathbf{3}}$ emerges at $\omega / \omega_{1}=1.285$ and first develops to the left towards decreasing frequencies. The branch is characterised at first by a strong coupling between modes 1 and 2 (visible in fig. 3(d)) and then by a coupling amongst modes 1,14 and 16. The order of the resonance can again be extrapolated from a Runge-Kutta time-domain scheme fed with the AUTO output. This gives fig. 4(b) and (c) where it is seen that modes 1 and 2 undergo a second 1:2 internal resonance, whereas modes 1-14 and 1-16 display a 1:10 internal resonance. Thus the dynamics of this branch is again dominated by even-order internal resonances. The last branch is $\mathbf{B}^{\mathbf{4}}$. This is an improper labelling because this branch is actually the principal branch undergoing an internal resonance with mode $11\left(\mathbf{B}_{11}^{4}\right.$, magenta). This branch is almost entirely unstable and the Runge-Kutta time domain simulation does not return stable periodic solutions. There is no doubt however that the branch is activated by internal resonance between modes 1 and 11, given the rapid growth of the latter in the bifurcation diagram at the expense of mode 1.

The analysis of the first NNM revealed some important aspects of the nonlinear system: in particular it was shown that the bifurcated branches are generated by even-order internal resonances which, in turn, break the symmetry of the cubic nonlinearity possessed by the system. This symmetry-breaking bifurcation has already been observed for the simple Duffing equation [31,40], as well as in systems with material nonlinearity [36]. Physically speaking, the most important properties returned by the analysis of the free NNM are: (i) the loss of stability of the periodic solutions for amplitudes above $3 h$; (ii) the pitchfork bifurcation giving rise to $\mathbf{B}^{2}$ presenting a strong coupling between modes 1 and 2. The next subsection will treat in some detail a few examples of forced-damped vibrations and it will be seen how the shape of the NNM gets modified by the damping and forcing terms.

\subsubsection{Forced-Damped Vibrations}

In this section forced-damped vibrations are considered. The plate is forced with a sinusoid of maximum amplitude $f$ and frequency $\Omega$ (see eq. (9)) varied around the eigenfrequency of the first mode, $\omega_{1}$. In turn, damping and forcing terms modify the shape of the invariant manifold corresponding to the NNM of the previous section. Internal resonances change too: some are basically unseen by the modified NNM, whereas others play a major role.

The first case under study presents a forcing amplitude of $f=0.17 \mathrm{~N}$, and a damping coefficient $\chi_{i}=0.001$ (same for all modes). The result is pictured in fig. 5. In the figure, the forced branches are represented with the usual colouring scheme (blue for mode 1 and red for mode 2) whereas the black lines are the branches from the Hamiltonian dynamics. The point labelled $\mathbf{G}$ in fig. 5 corresponds to a pitchfork symmetry-breaking bifurcation, driven by the underlying Hamiltonian dynamics and by the existence of the 1:2 internal resonance. The main branch becomes unstable in favour of stable periodic orbits where both modes 1 and 2 are activated in a 1:2 internal resonance. Hence branch $\mathbf{B}^{2}$ reveals its importance as it has a major effect in the damped-driven case. One can also notice that, for this small amount of damping, the turning point $\mathbf{J}$ is located just before the resonant tongue along the original backbone curve.

In order to understand more deeply the role of the branch $\mathbf{B}^{2}$, two more cases of interest are portrayed in fig. 6 and fig. 7. Here $f=1.36 \mathrm{~N}$ for both cases, and $\chi_{i}=0.005$ for fig. 6 and 0.001 for fig. 7 . The first important remark is the location of the pitchfork bifurcation along the main branch: $q_{1} / h=1.899$ for fig. 6 and $q_{1} / h=1.824$ for fig. 7 . It is seen that the invariant manifold of the Hamiltonian dynamics is largely affected by the damping and forcing terms: the bifurcation $\mathbf{G}$ is located at very different points in the phase space when comparing free and forced-damped vibrations. The 1:2 internal resonance giving rise to $\mathbf{B}^{\mathbf{2}}$ becomes in the latter case a dominant part of the dynamics, taking up a large portion of the phase space composed mainly of stable solutions. As a consequence, stable solutions are found on $\mathbf{B}^{2}$ at amplitudes larger then $3 h$. In addition, there is no trace of the other bifurcations giving rise to $\mathbf{B}^{\mathbf{3}}, \mathbf{B}^{\mathbf{4}}$ in the Hamiltonian dynamics. This observation leads to the conclusion that the free and forced-damped analyses are complementary: on one hand, it is not straightforward to understand which bifurcations are key to the forced-damped vibrations when looking solely at the Hamiltonian dynamics; on the other hand, the forced-damped system is more easily interpreted by making use of the free vibrations diagrams. Hence, a complete scenario for the forced-damped vibrations cannot be obtained if a preliminary analysis of free vibrations is disregarded. 

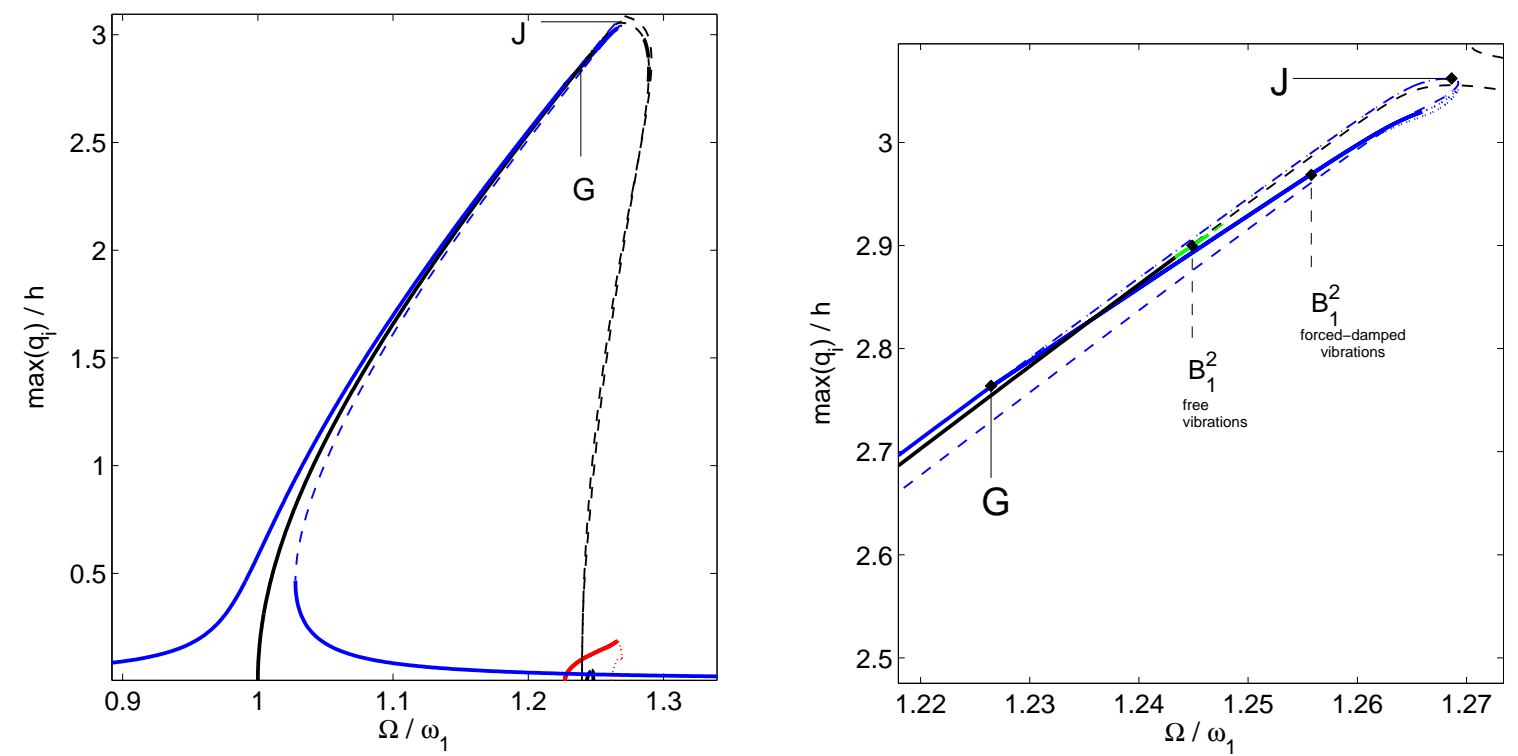

Fig. 5 Forced response for mode 1 with $f=0.17 \mathrm{~N}, \chi=0.001$. G: pitchfork bifurcation point leading to the coupled solution; $\mathbf{J}$ turning point. Mode 1: blue, Mode 2: red.
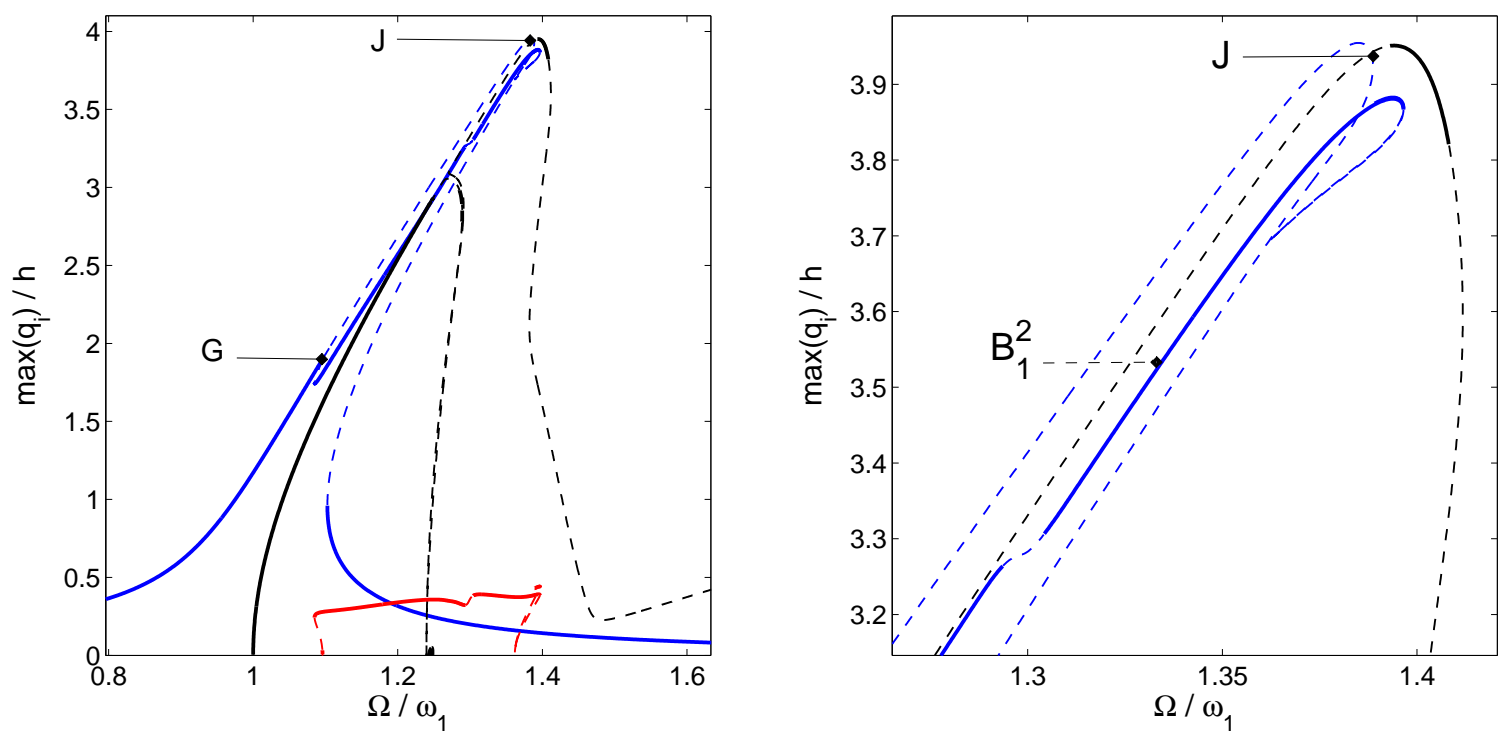

Fig. 6 Forced response for mode 1 with $f=1.36 \mathrm{~N}, \chi=0.005$. G: pitchfork bifurcation point leading to the coupled solution; $\mathbf{J}$ turning point. Mode 1: blue, Mode 2: red.

\subsection{Mode 2}

\subsubsection{Free Vibrations}

Fig. 8 shows the second NNM for $N_{w}=16$. Convergence in this case is not shown for the sake of brevity; note however that the convergence study gave results comparable to those of mode 1 . Thus the same model including $N_{w}=16$ modes is kept for the remainder of the study. Once again, one can notice that no stable solutions are found beyond a certain amplitude limit, which is numerically found at $1.5 \mathrm{~h}$ for mode 2 . Actually, the principal branch loses its stability at the appearance of the coupled branch. As for mode 1, some modes are activated by nonresonant coupling, and these are the

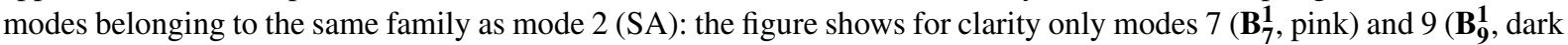
blue). The most salient feature of the dynamics is the internal resonance between modes 2 and 5: a time integration was performed on $\mathbf{B}^{2}$ at $\omega / \omega_{1}=2.0515$, leading to the solution visible in the inset of fig. 8 showing a 1:2 internal resonance. Interestingly, this branch is almost entirely unstable, except on the interval $2.051 \leq \omega / \omega_{1} \leq 2.052$. As for mode 1 , the Hamiltonian manifold will be modified when damping and forcing are introduced in the system. 

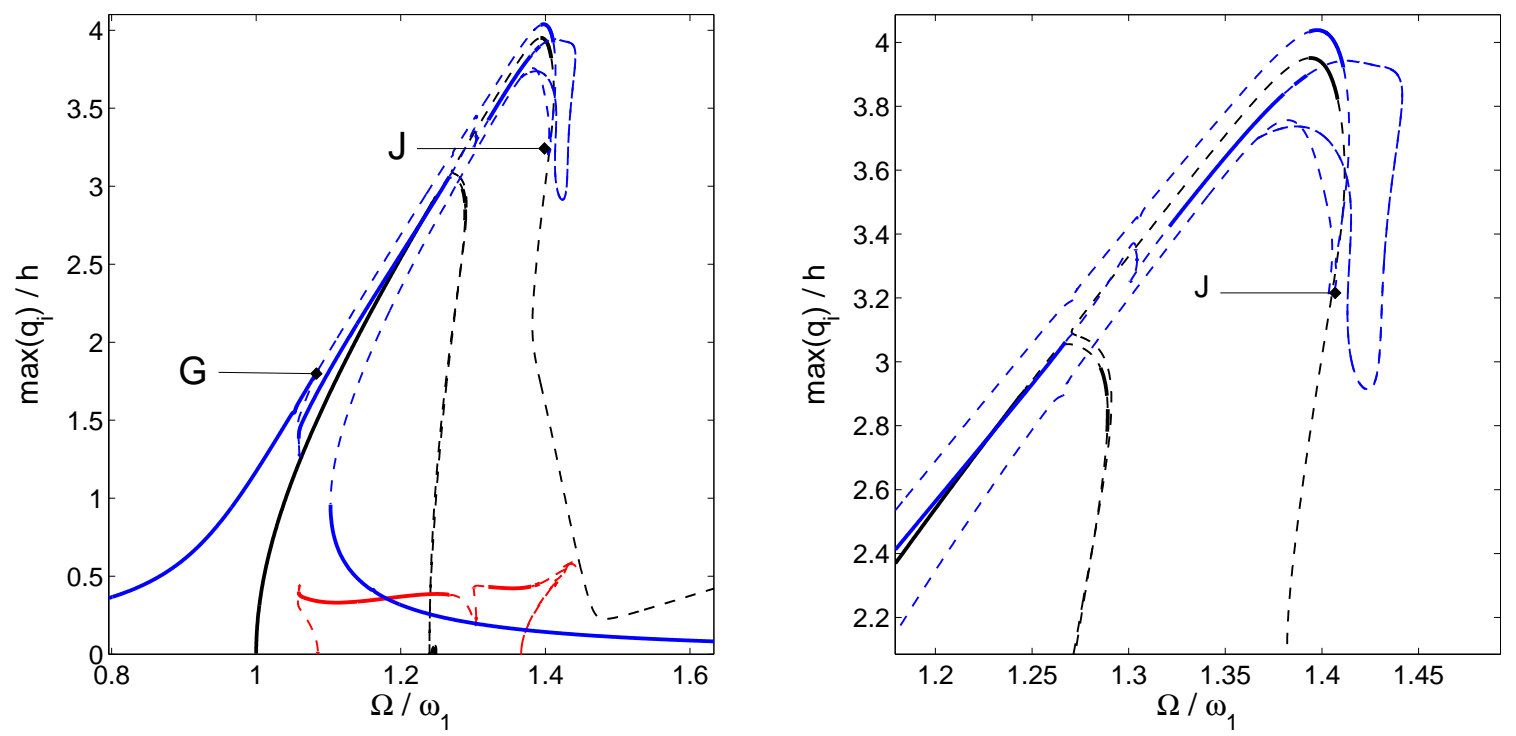

Fig. 7 Forced response for mode 1 with $f=1.36 \mathrm{~N}, \chi=0.001$. G: pitchfork bifurcation point leading to the coupled solution; $\mathbf{J}$ turning point. Mode 1: blue, Mode 2: red.

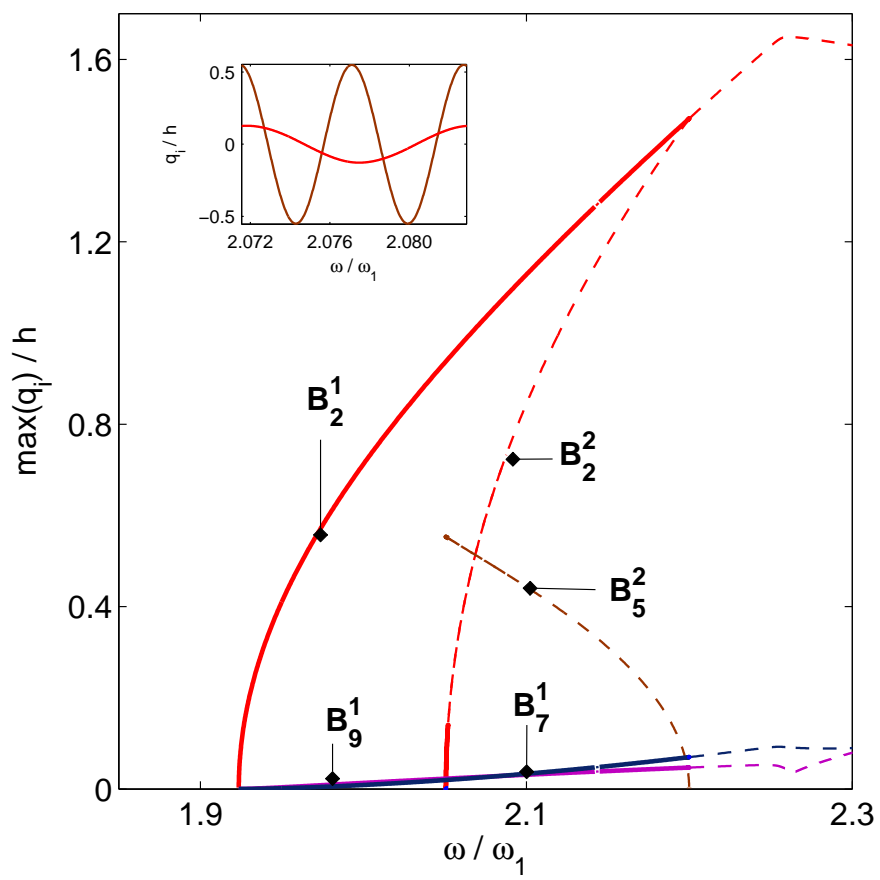

Fig. 8 Backbone for mode 2 obtained when $N_{w}=16$. Modes 7 (pink) and 9 (dark blue) are activated by the nonresonant coupling within the SA family; mode 5 (brown) from the AA family is activated by 1:2 internal resonance (see inset).

\subsubsection{Forced-Damped Vibrations}

Examples of forced-damped solution are presented in fig. 9. The cases (a) and (b) present the same damping coefficient, $\chi_{i}=0.001$, and the forcing values are, respectively, $f=1.2 \mathrm{~N}, f=2.0 \mathrm{~N}$. Both forcing values are sufficient to reach amplitudes high enough to detect the internal resonance with mode 5. For case (a) the bifurcated branch remains almost completely unstable, as for the Hamiltonian dynamics. When the forcing is high enough, however, stable solutions appear along the interval $2.2 \leq \omega / \omega_{1} \leq 2.3$. As a consequence, mode 2 possesses a secondary branch of stable periodic orbits of amplitude greater than $1.5 \mathrm{~h}$, which was seen to be the limit of stability for the Hamiltonian manifold. As for mode 1, it is seen that the introduction of forcing and damping may lead to extended stable solutions on the coupled branches. Another case of interest is portrayed in fig.9(c). Here the maximum forcing is $f=3.2 \mathrm{~N}$ and the damping coefficient is $\chi_{i}=0.01$. In this case the damping effects are so evident that the turning point is located away from the backbone. 

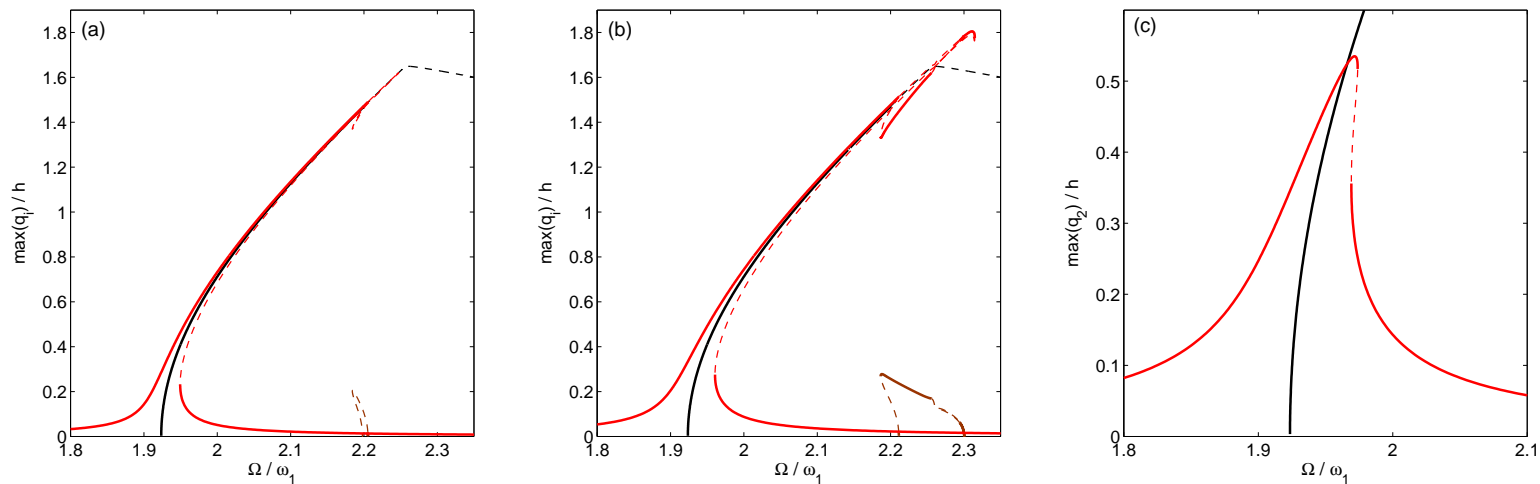

Fig. 9 Examples of forced-damped vibrations around the NNM for mode 2. (a): $f=1.2 \mathrm{~N}, \chi=0.001 ;$ (b): $f=2.0 \mathrm{~N}, \chi=0.001 ;$ (c): $f=3.2$ $\mathrm{N}, \chi=0.01$. Mode 2: red, Mode 5: brown.

Distortion is a typical effect of damping: the forced response does not fit tightly along the backbone and the turning point moves away from it.

In turn, the analysis of the forced responses for mode 1 and 2 revealed some interesting aspects of the global dynamics: (i) symmetry breaking resonances are common and key to the dynamics of the dynamical response; (ii) stable solutions on the coupled branches may reach higher amplitudes than the Hamiltonian manifold, for particular combinations of damping and forcing factors.

\section{Conclusions}

The nonlinear dynamics of rectangular plates has been investigated. A robust numerical method has been developed to obtain accurate modal solutions for a very large number of modes. In this sense, a fast converging solution strategy has been derived for the calculation of the eigenmodes of a fully clamped plate (needed here to solve for the Airy stress function of a plate in a nonlinear regime). Formal symmetry properties and coupling rules have been illustrated to allow large computational and memory savings when calculating the coupling coefficients $\Gamma$ 's. Reference values for some of these coefficients, previously unavailable in the case of a rectangular geometry, have been presented.

Free and forced vibrations have then been taken under consideration for the first two modes. For the first time, the NNM branches of solution (conservative case) have been drawn out to very large amplitudes, showing the existence of internal resonance branches. An important feature, the nonexistence of periodic solutions beyond some vibration amplitude ( $4 h$ for mode $1,1.8 h$ for mode 2 ) has been found. A thorough comparison of the Hamiltonian dynamics with the forced-damped (observable) dynamics has been derived, in order to highlight: (i) the necessity of a preliminary analysis of the free vibrations, (ii) the main differences one can expect between the NNMs of the conservative systems and the observable periodic orbits of the forced-damped system. Simple features such as the shift of the turning point from the backbone for large values of the damping, have been found. More interestingly, the importance of certain internal resonance tongues (those with the simpler ratio) has been underlined, whereas other are mostly undetected in the forced case. Finally it has been found that some coupled branches may override the amplitude limit of existence of periodic solutions predicted by the backbone curve.

Even though the results presented here involve at most 16 modes, the numerical scheme developed is able to consider a few hundred of them interacting together. The results shown here have been necessary to validate the model, which will be used to undertake further study of more involved dynamical problems (i.e. wave turbulence or sound synthesis of damped impacted plates for the reproduction of gong-like sounds). 


\section{References}

1. M. Amabili. Nonlinear vibrations of rectangular plates with different boundary conditions: theory and experiments. Computers and Structures, 82(31-32):2587-2605, 2004.

2. M. Amabili. Nonlinear Vibrations and Stability of Shells and Plates. Cambridge University Press, 2008.

3. G. Anlas and O. Elbeyli. Nonlinear vibrations of a simply supported rectangular metallic plate subjected to transverse harmonic excitation in the presence of a one-to-one internal resonance. Nonlinear Dynamics, 30(1):1-28, 2002.

4. J. Awrejcewicz, V. A. Krysko, and A. V. Krysko. Spatio-temporal chaos and solitons exhibited by von Kármán model. I. J. Bifurcation and Chaos, pages 1465-1513, 2002.

5. S. Bilbao. A family of conservative finite difference schemes for the dynamical von Kármán plate equations. Numerical Methods for Partial Differential equations, 24(1):193-216, 2008.

6. S. Bilbao. Numerical Sound Synthesis: Finite Difference Schemes and Simulation in Musical Acoustics. Wiley, 2009.

7. S. Bilbao. Percussion synthesis based on models of nonlinear shell vibration. IEEE Trans. Audio, Speech and Lang. Proc., 18(4):872-880, May 2010.

8. F. Blanc, C. Touzé, J.-F. Mercier, K. Ege, and A.-S. Bonnet Ben-Dhia. On the numerical computation of nonlinear normal modes for reduced-order modelling of conservative vibratory systems. Mechanical Systems and Signal Processing, (0):-, 2012.

9. A. Boudaoud, O. Cadot, B. Odille, and C. Touzé. Observation of wave turbulence in vibrating plates. Phys. Rev. Lett., 100:234504, Jun 2008.

10. F. Boumediene, L. Duigou, E.H. Boutyour, A. Miloudi, and J.M. Cadou. Nonlinear forced vibration of damped plates by an asymptotic numerical method. Computers and Structures, 87(23-24):1508-1515, 2009.

11. A. Chaigne and C. Lambourg. Time-domain simulation of damped impacted plates. I. theory and experiments. The Journal of the Acoustical Society of America, 109(4):1422-1432, 2001.

12. A. Chaigne, C. Touzé, and O. Thomas. Nonlinear vibrations and chaos in gongs and cymbals. Acoustical Science and Technology, 26:403-409, 2005.

13. S.I. Chang, A.K. Bajaj, and C.M. Krousgrill. Nonlinear oscillations of a fluttering plate. AIAA Journal, 4:1267-1275, July 1966.

14. S.I. Chang, A.K. Bajaj, and C.M. Krousgrill. Non-linear vibrations and chaos in harmonically excited rectangular plates with one-to-one internal resonance. Nonlinear Dynamics, 4:433-460, 1993.

15. W. Q. Chen and H. J. Ding. On free vibration of a functionally graded piezoelectric rectangular plate. Acta Mechanica, 153(3-4):207-216, 2002 .

16. C.Y. Chia. Nonlinear Analysis of Plates. Mc Graw Hill, New York, 1980.

17. H.N. Chu and G. Herrmann. Influence of large amplitudes on free flexural vibrations of rectangular elastic plates. Journal of Applied Mechanics, 23, 1956.

18. O. Doaré and S. Michelin. Piezoelectric coupling in energy-harvesting fluttering flexible plates: linear stability analysis and conversion efficiency. Journal of Fluids and Structures, 27:1357-1375, November 2011.

19. E. Doedel, R.C. Paffenroth, A.R. Champneys, T.F. Fairgrieve, Y.A. Kuznetsov, B.E. Oldeman, B. Sandstede, and X Wang. Auto2000: Continuation and bifurcation software for ordinary differential equations (with homcont). Technical report, Concordia University, Canada, 2002.

20. G. Düring, C. Josserand, and S. Rica. Weak turbulence for a vibrating plate: Can one hear a kolmogorov spectrum? Phys. Rev. Lett., 97:025503, Jul 2006.

21. Y. M. Fu and C. Y. Chia. Nonlinear bending and vibration of symmetrically laminated orthotropic elliptical plate with simply supported edge. Acta Mechanica, 74(1-4):155-170, 1988.

22. Y. Gao, B. Xu, and H. Huh. Electromagneto-thermo-mechanical behaviors of conductive circular plate subject to time-dependent magnetic fields. Acta Mechanica, 210(1-2):99-116, 2010.

23. M. Géradin and D. Rixen. Mechanical Vibrations. John Wiley and Sons, 1997.

24. R.E. Gordnier and M.R. Visbal. Development of a three-dimensional viscous aeroelastic solver for nonlinear panel flutter. Journal of Fluids and Structures, 16(4):497 - 527, 2002.

25. P. Hagedorn and A. DasGupta. Vibrations and Waves in Continuous Mechanical Systems. John Wiley and Sons, Chichester, UK, 2007.

26. G. Kerschen, M. Peeters, J.C. Golinval, and A.F. Vakakis. Nonlinear normal modes, part I: A useful framework for the structural dynamicist. Mechanical Systems and Signal Processing, 23(1):170 - 194, 2009.

27. G. C. Kung and Y.-H. Pao. Nonlinear flexural vibrations of a clamped circular plate. Journal of Applied Mechanics, 39(4):1050-1054, 1972.

28. K. A. Legge and N. H. Fletcher. Nonlinearity, chaos, and the sound of shallow gongs. The Journal of the Acoustical Society of America, 86(6):2439-2443, 1989.

29. A. Leissa. Vibration of plates. Acoustical Society of America, 1993.

30. W.L. Li. Vibration analysis of rectangular plates with general elastic support. Journal of Sound and Vibration, 273(3):619-635, 2003.

31. A.C.J Luo and J. Huang. Analytical solutions for asymmetric periodic motions to chaos in a hardening duffing oscillator. Nonlinear Dynamics, 72(1-2):417-438, 2013.

32. J.C. Golinval C. Stephan P. Lubrina M. Peeters, G. Kerschen. Nonlinear normal modes of a full-scale aircraft. In 29th International Modal Analysis Conference, Jacksonville (USA), 2011.

33. J. Meenen and H. Altenbach. A consistent deduction of von Kármán-type plate theories from three-dimensional nonlinear continuum mechanics. Acta Mechanica, 147(1-4):1-17, 2001.

34. N. Mordant. Are there waves in elastic wave turbulence? Phys. Rev. Lett., 100:234505, Jun 2008.

35. N. Mordant. Fourier analysis of wave turbulence in a thin elastic plate. The European Physical Journal B, 76:537-545, 2010.

36. M.O. Moussa, Z. Moumni, O. Doaré, C. Touzé, and W. Zaki. Non-linear dynamic thermomechanical behaviour of shape memory alloys. Journal of Intelligent Material Systems and Structures, 23(14):1593-1611, 2012.

37. K.D. Murphy, L.N. Virgin, and S.A. Rizzi. Characterizing the dynamic response of a thermally loaded, acoustically excited plate. Journal of Sound and Vibration, 196(5):635 - 658, 1996.

38. A.H. Nayfeh. Nonlinear Oscillations. John Wiley and Sons, 1995.

39. A.H. Nayfeh and P.F. Pai. Linear and Nonlinear Structural Mechanics. John Wiley and Sons, 2004.

40. U. Parlitz and W. Lauterborn. Superstructure in the bifurcation set of the duffing equation. Physics Letters A, 107(8):351 - 355, 1985 .

41. M. Peeters, R. Viguié, G. Sérandour, G. Kerschen, and J.-C. Golinval. Nonlinear normal modes, part II: Toward a practical computation using numerical continuation techniques. Mechanical Systems and Signal Processing, 23(1):195 - 216, 2009.

42. P. Ribeiro. Nonlinear vibrations of simply-supported plates by the p-version finite element method. Finite Elements in Analysis and Design, 41(9-10):911-924, 2005.

43. P. Ribeiro and M. Petyt. Geometrical non-linear, steady-state, forced, periodic vibration of plate, part I: model and convergence study. Journal of Sound and Vibration, 226(5):955-983, 1999.

44. P. Ribeiro and M. Petyt. Geometrical non-linear, steady-state, forced, periodic vibration of plate, part II: stability study and analysis of multimodal response. Journal of Sound and Vibration, 226(5):985-1010, 1999. 
45. M. Sathyamoorthy. Nonlinear vibrations of plates: An update of recent research developments. Applied Mechanics Reviews, 49(10S):S55S62, 1996.

46. O. Thomas and S. Bilbao. Geometrically nonlinear flexural vibrations of plates: In-plane boundary conditions and some symmetry properties. Journal of Sound and Vibration, 315(3):569-590, 2008.

47. O. Thomas, C. Touzé, and A. Chaigne. Non-linear vibrations of free-edge thin spherical shells: modal interaction rules and 1:1:2 internal resonance. International Journal of Solids and Structures, 42(1112):3339 - 3373, 2005.

48. J.J. Thomsen. Vibrations and Stability. Springer, 2003.

49. C. Touzé, S. Bilbao, and O. Cadot. Transition scenario to turbulence in thin vibrating plates. Journal of Sound and Vibration, 331(2):412433, 2011.

50. C. Touzé, O. Thomas, and M. Amabili. Transition to chaotic vibrations for harmonically forced perfect and imperfect circular plates. International Journal of Non-Linear Mechanics, 46(1):234 - 246, 2011.

51. C. Touzé, O. Thomas, and A. Chaigne. Hardening/softening behaviour in non-linear oscillations of structural systems using non-linear normal modes. Journal of Sound and Vibration, 273(1-2):77 - 101, 2004.

52. C. Touzé, O. Thomas, and A. Huberdeau. Asymptotic non-linear normal modes for large-amplitude vibrations of continuous structures. Computers \& structures, 82(31):2671-2682, 2004.

53. A.F. Vakakis. Non-linear normal modes (nnms) and their applications in vibration theory: An overview. Mechanical Systems and Signal Processing, 11(1):3 - 22, 1997.

54. T. von Kármán. Festigkeitsprobleme im maschinenbau. Encyklopadie der Mathematischen Wissenschaften, 4:311-385, 1910.

55. N. Yamaki. Influence of large amplitudes on flexural vibrations of elastic plates. Zeitschrift für Angewandte Mathematik und Mechanik, 41(12):501-510, 1961.

56. X.L. Yang and P.R. Sethna. Local and global bifurcations in parametrically excited vibrations of nearly square plates. International Journal of Non-Linear Mechanics, 26(2):199 - 220, 1991.

\section{A Matrices for the Clamped Plate Problem}

To set up the eigenvalue problem, eq. (25), one may proceed as follows. First, it is necessary to define the size of the square matrices $K_{i j}, M_{i j}$. Suppose this size is $A^{2} \times A^{2}$ (where $\mathrm{A}$ is an integer). Then, the indices $n_{1}, n_{2}$ for the expansion function (22) range from 0 to $A-1$. In this way, the total number of eigenvalues calculated will be $A^{2}$. Note that all the quantities that appear in the definition of the matrices are quadratic, so one needs really four indices to define the ${ }_{i j}$ entry in each matrix. Suppose these indices are $(m, n)$ and $(p, q)$. Then

$$
\begin{aligned}
& K(i, j)=K(m n, p q)=\int_{0}^{L_{x}} X_{m}^{\prime \prime}(x) X_{p}^{\prime \prime}(x) d x \int_{0}^{L_{y}} Y_{n}(y) Y_{q}(y) d y+\int_{0}^{L_{x}} X_{m}(x) X_{p}(x) d x \int_{0}^{L_{y}} Y_{n}^{\prime \prime}(y) Y_{q}^{\prime \prime}(y) d y+2 \int_{0}^{L_{x}} X_{m}^{\prime}(x) X_{p}^{\prime}(x) d x \int_{0}^{L_{y}} Y_{n}^{\prime}(y) Y_{q}^{\prime}(y) d y \\
& M(i, j)=M(m n, p q)=\int_{0}^{L_{x}} X_{m}(x) X_{p}(x) d x \int_{0}^{L_{y}} Y_{n}(y) Y_{q}(y) d y
\end{aligned}
$$

The integrals are

$$
\begin{aligned}
& \int_{0}^{L_{x}} X_{m}^{\prime \prime}(x) X_{p}^{\prime \prime}(x) d x= \\
& \begin{cases}720 / L_{x}^{3} ; & \text { if } m=p=0 \\
\left(\pi^{4} m^{4}-672(-1)^{m}-768\right) /\left(2 L_{x}^{3}\right) ; & \text { if } m=p \neq 0 \\
0 & \text { if } m \text { or } p=0 \text { and } m \neq p \\
-24\left(7(-1)^{m}+7(-1)^{p}+8(-1)^{m}(-1)^{p}+8\right) / L_{x}^{3} ; \text { otherwise } & \end{cases} \\
& \begin{array}{ll}
\int_{0}^{L_{x}} X_{m}(x) X_{p}(x) d x= & \text { if } m=p=0
\end{array} \\
& \begin{cases}10 L_{x} / 7 ; & \text { if } m=p \neq 0 \\
67 L_{x} / 70-(-1)^{m} L_{x} / 35-768 L_{x} /\left(\pi^{4} m^{4}\right)-672(-1)^{m} L_{x} /\left(\pi^{4} m^{4}\right) ; & \text { if } p=0 \text { and } p \neq 0 \\
\left.3 L_{x}\left((-1)^{p}+1\right)\left(\pi^{4} p^{4}-1680\right)\right) /\left(14 \pi^{4} p^{4}\right) ; & \\
\left.3 L_{x}\left((-1)^{m}+1\right)\left(\pi^{4} m^{4}-1680\right)\right) /\left(14 \pi^{4} m^{4}\right) ; & \\
-\left(L _ { x } \left(11760(-1)^{m}+11760(-1)^{p}-16 \pi^{4} m^{4}+13440(-1)^{m}(-1)^{p}+\right.\right. & \\
\left.\left.(-1)^{m} \pi^{4} m^{4}+(-1)^{p} \pi^{4} m^{4}-16(-1)^{m}(-1)^{p} \pi^{4} m^{4}+13440\right)\right) /\left(70 \pi^{4} m^{4}\right) & \\
-\left(L_{x}\left(13440 m^{4}+11760(-1)^{m} m^{4}+11760(-1)^{p} m^{4}+13440(-1)^{m}(-1)^{p} m^{4}\right)\right) /\left(70 \pi^{4} m^{4} p^{4}\right) ; \text { otherwise }\end{cases}
\end{aligned}
$$$$
\int_{0}^{L_{x}} X_{m}^{\prime}(x) X_{p}^{\prime}(x) d x=
$$

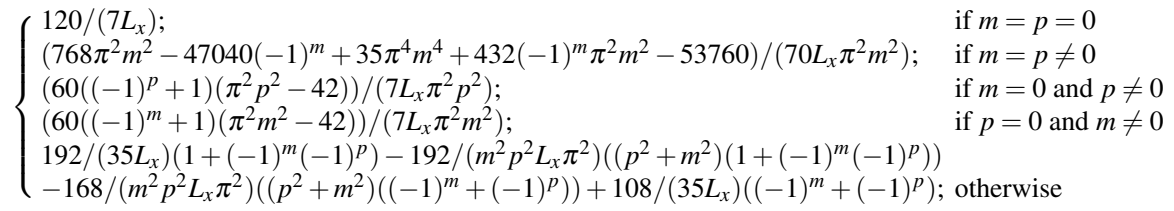

and similarly for the integrals involving the functions $Y$. 